OPEN ACCESS

Edited by: Joao P. B. Viola, Instituto Nacional de Câncer (INCA), Brazil

Reviewed by: Raymond P. Donnelly, United States Food and Drug Administration, United States

Howard M. Johnson, University of Florida, United States

*Correspondence: Hans A. R. Bluyssen

h.bluyss@amu.edu.p

Specialty section: This article was submitted to Molecular Innate Immunity, a section of the journal Frontiers in Immunology

Received: 19 March 2018 Accepted: 07 May 2018 Published: 28 May 2018

Citation:

Michalska A, Blaszczyk K, Wesoly $J$ and Bluyssen HAR (2018) A Positive

Feedback Amplifier Circuit That Regulates Interferon (IFN)-Stimulated Gene Expression and Controls Type I and Type II IFN Responses.

Front. Immunol. 9:1135 doi: 10.3389/fimmu.2018.01135

\section{A Positive Feedback Amplifier Circuit That Regulates Interferon (IFN)-Stimulated Gene Expression and Controls Type I and Type II IFN Responses}

\author{
Agata Michalska ${ }^{1}, K^{2}$ atarzyna Blaszczyk ${ }^{1}$, Joanna Wesoly ${ }^{2}$ and Hans A. R. Bluyssen ${ }^{1 \star}$
}

${ }^{1}$ Department of Human Molecular Genetics, Faculty of Biology, Institute of Molecular Biology and Biotechnology, Adam Mickiewicz University, Poznan, Poland, ${ }^{2}$ Laboratory of High Throughput Technologies, Faculty of Biology, Institute of Molecular Biology and Biotechnology, Adam Mickiewicz University, Poznan, Poland

Interferon (IFN)-I and IFN-II both induce IFN-stimulated gene (ISG) expression through Janus kinase (JAK)-dependent phosphorylation of signal transducer and activator of transcription (STAT) 1 and STAT2. STAT1 homodimers, known as $\gamma$-activated factor (GAF), activate transcription in response to all types of IFNs by direct binding to IFN-II activation site ( $\gamma$-activated sequence)-containing genes. Association of interferon regulatory factor (IRF) 9 with STAT1-STAT2 heterodimers [known as interferon-stimulated gene factor 3 (ISGF3)] or with STAT2 homodimers (STAT2/IRF9) in response to IFN-I, redirects these complexes to a distinct group of target genes harboring the interferon-stimulated response element (ISRE). Similarly, IRF1 regulates expression of ISGs in response to IFN-I and IFN-II by directly binding the ISRE or IRF-responsive element. In addition, evidence is accumulating for an IFN-independent and -dependent role of unphosphorylated STAT1 and STAT2, with or without IRF9, and IRF1 in basal as well as long-term ISG expression. This review provides insight into the existence of an intracellular amplifier circuit regulating ISG expression and controlling long-term cellular responsiveness to IFN-I and IFN-II. The exact timely steps that take place during IFN-activated feedback regulation and the control of ISG transcription and long-term cellular responsiveness to IFN-I and IFN-II is currently not clear. Based on existing literature and our novel data, we predict the existence of a multifaceted intracellular amplifier circuit that depends on unphosphorylated and phosphorylated ISGF3 and GAF complexes and IRF1. In a combinatorial and timely fashion, these complexes mediate prolonged ISG expression and control cellular responsiveness to IFN-I and IFN-II. This proposed intracellular amplifier circuit also provides a molecular explanation for the existing overlap between IFN-I and IFN-II activated ISG expression.

Keywords: interferon, JAK/signal transducer and activator of transcription signaling pathway, signal transducer and activator of transcriptions, interferon-stimulated gene factor 3 , interferon regulatory factor 1 , transcriptional regulation, antiviral activity

\section{INTRODUCTION}

Interferons (IFNs) belong to the superfamily of cytokines that were discovered by Isaacs and Lindenmann as antiviral proteins (1). Since then it has become clear that IFNs do much more than inhibiting virus replication, and are also involved in cell proliferation, apoptosis, inflammation, as well as adaptive immunity $(2-4)$. 
Interferons comprise a family of molecules divided into three main sub families: IFN-I, IFN-II, and IFN-III. IFN-II is also known as IFN $\gamma$ that binds the IFN $\gamma$ receptor (IFNGR) complex and mediates broad immune responses to non-viral pathogens. IFN-II is mainly produced in response to foreign antigens or mitogens by $\mathrm{T}$ lymphocytes and natural killer (NK) cells. IFN-I predominantly consists of IFN $\alpha$ and IFN $\beta$ subtypes and can be produced by many substances in a variety of cell types. However, viruses and synthetic double-stranded RNAs are the most potent inducers of IFN-I. They engage the ubiquitously expressed IFN $\alpha$ receptor (IFNAR) complex and are known to be crucial for activating a robust host response against viral infection (2-4). IFN-III contains the subtypes IFN $\lambda 1$, IFN $\lambda 2$, IFN $\lambda 3$ (5), and the recently discovered IFN $\lambda 4$ (6). These IFNs signal through a receptor complex consisting of IL10R2 and IFNLR1 and possess potent antiviral activity (5).

Interferon-I and IFN-II both induce IFN-stimulated gene (ISG) expression through Janus kinase (JAK)-dependent phosphorylation of signal transducer and activator of transcription (STAT) 1 and STAT2. STAT1 homodimers, known as $\gamma$-activated factor (GAF), activate transcription in response to all types of IFNs by direct binding to IFN-II activation site $[\gamma$-activated sequence (GAS)]-containing genes. Association of interferon regulatory factor (IRF) 9 with STAT1-STAT2 heterodimers [known as interferon-stimulated gene factor 3 (ISGF3)] or with STAT2 homodimers (STAT2/IRF9) in response to IFN-I, redirects these complexes to a distinct group of target genes harboring the interferon-stimulated response element (ISRE) (7). Similarly, IRF1 can regulate expression of ISGs in response to IFN-I and IFN-II by directly binding the ISRE or IRF-responsive element (IRE) $(8,9)$. The partially overlapping and differential activation of transcription factor complexes and regulation of target gene expression by IFN-I and IFN-II, may be a consequence of the biological similarities and differences of these two types of IFN.

According to the general paradigm, phosphorylation of STAT1 and STAT2 in response to IFN-I and or IFN-II displays a robust and transient character. This is followed by a similar ISG expression pattern that decreases over time. However, recent studies have shown that IFN signaling is much more complex and revealed that ISG expression patterns are globally sustained in response to both types of IFN (10-12). This sustained response relies on prolonged expression of the ISGF3 and GAF components STAT1, STAT2 and IRF9 and IRF1 as part of a positive feedback loop. In addition, evidence is accumulating for a role of U-STAT1 and U-STAT2, with or without IRF9, and IRF1 in basal as well as long-term ISG expression.

This review combines our latest findings with recent literature to provide insight into the existence of an intracellular amplifier circuit regulating ISG expression and controlling cellular responsiveness to IFN-I and IFN-II. Especially, we focus on how this feedback system regulates ISG transcription from the basal to the IFN-induced state at the genome-wide level, how it depends on phosphorylation and expression of ISGF3, IRF1, and GAF components, and how it controls cellular responsiveness to IFN-I and IFN-II in relation to antiviral activity.

\section{PHOSPHORYLATED STAT1- AND STAT2-DEPENDENT ISG TRANSCRIPTION: ISGF3, STAT2/IRF9, AND GAF}

\section{ISGF3: Phosphorylated STAT1 and STAT2 with IRF9}

All IFN-I subtypes bind the IFNAR1 and IFNAR2 subunits of the heterodimeric transmembrane IFNAR receptor to activate the JAK/STAT pathway, used by many cytokines and growth factors. The main constituents of this pathway are: (I) the JAK family of non-receptor tyrosine kinases JAK1, JAK2, JAK3, and tyrosine kinase 2 (TYK2) (13) and (II) transcription factors of the STAT family STAT1-STAT6. STATs are characterized by seven structurally and functionally conserved regions: the N-terminal domain, coiled-coil domain, DNA-binding domain, linker domain, Src-homology 2 domain, tyrosine phosphorylation site, and transcriptional activation domain (13-15).

Interferon-I binding to IFNAR results in receptor dimerization and increased JAK1 and TYK2 kinase activity via juxtapositioning and transphosphorylation (13). Subsequently, JAK1 and TYK2 phosphorylate IFNAR1 and IFNAR2 on target tyrosine residues that become docking sites for STAT1 and STAT2 (14). Receptor-bound STAT1 and STAT2 are thus phosphorylated on a critical tyrosine residue (pTyr) driving SH2-pTyr mediated dimer formation, nuclear translocation, and transcriptional activation. In the canonical pathway of IFN-I-mediated signaling, Tyr701 phosphorylation of STAT1 and Tyr690 of STAT2 leads to heterodimerization, interaction with IRF9 and formation of ISGF3 (Figure 1). After translocation to the nucleus, this complex binds the ISRE (consensus sequence AGTTTCN 2 TTTCN) of over 300 ISGs, such as ISG15, OAS1-3, IFIT1-3, or MX1 and 2 that are instrumental in antiviral activity (13-15) (Figure 1).

The basic function of the ISGF3-dependent response is to mediate rapid and robust IFN-I responses by regulating transient transcription of antiviral ISGs (16). This fast and large-scale response enables to combat with infection, but simultaneously prevents long-term harmful effects to activated cells. For this reason, the ISGF3-dependent response is in general time-limited following a quick assembly of the complex from its pre-existing components and its transport to the nucleus where it binds to ISRE-containing ISGs. In this respect, STAT2 is constantly imported to the nucleus in an unphosphorylated state due to its association with IRF9 that contains a strong nuclear localization signal (NLS). The dominant nuclear export signal (NES) of STAT2 shuttles the complex back to the cytoplasm. Following STAT2 tyrosine phosphorylation, it can form dimers with STAT1 and the trimeric ISGF3 complex, and together with the NLS and NES present in STAT1 nucleocytoplasmic shuttling of ISGF3 and its components is controlled in a timely and spatial fashion (17). In 1989, Levy et al. provided evidence that the active ISGF3 complex is already detectable within 2 min after exposure of cells to IFN $\alpha$ (18). Thus, the rapid ISGF3 assembly serves fast and robust IFN $\alpha$ responses that are diminished in time and coincide with the phosphorylation profiles of STAT1 and STAT2 (19). In this process, important negative feedback mechanisms collaborate to dampen STAT phosphorylation and ISG expression several hours 


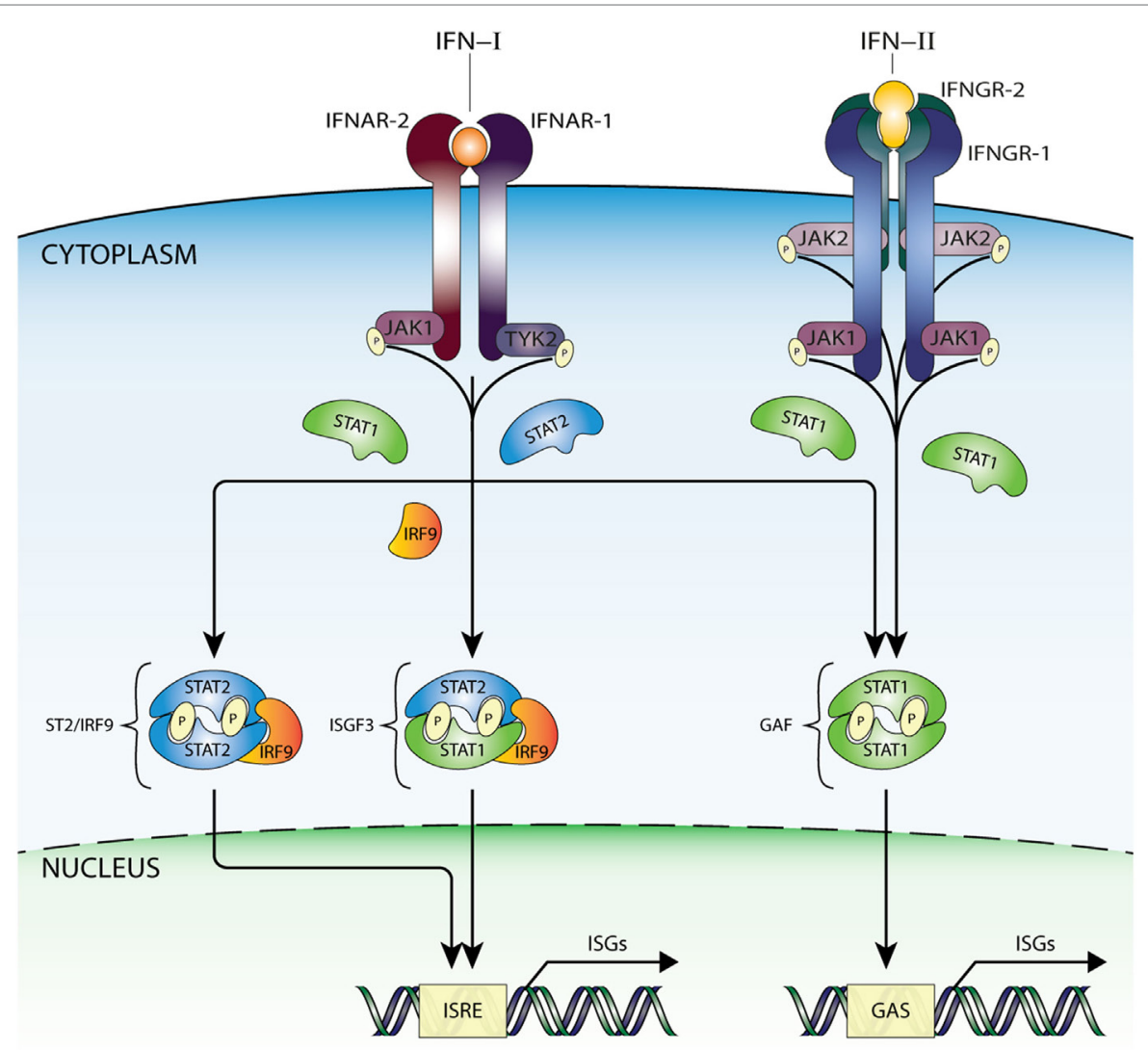

FIGURE 1 | IFN-activated ISG transcription mediated by ISGF3, GAF, and STAT2/IRF9 complexes. IFN-I is recognized by a heterodimeric receptor composed of IFNAR1 and IFNAR2 subunits. After IFN binding and receptor dimerization, juxtapositioning of JAK1 and TYK2 results in increased kinase activity via transphosphorylation and subsequent STAT protein recruitment. Receptor-bound STAT proteins are successively phosphorylated, dimerize, and translocate to nucleus, where ISG transcription is initiated after binding ISRE or GAS sites. Thus, in response to IFN-I three active complexes are formed that play a crucial role in transcriptional regulation. A STAT1/STAT2 heterodimer associated with IRF9, known as ISGF3, binds the ISRE motif present in >300 ISGs. Second, with the same mode of action, an alternative complex built of STAT2 homodimers and IRF9 (STAT2/IRF9). In addition, STAT1 homodimers (known as GAF), which specifically recognize the GAS sequence. On the other hand, IFN-II interacts with a different receptor built of two IFNGR1 and two IFNGR2 subunits connected with JAK1 and JAK2 kinases, which are capable of phosphorylating only STAT1 proteins, resulting in dimerization and formation of GAF. GAF translocates to the nucleus and targets GAS-containing genes, in a similar way as in response to IFN-I. Abbreviations: IFN, interferon; STAT, signal transducer and activator of transcription; IRF, interferon regulatory factor; JAK, Janus kinase; TYK, tyrosine kinase; ISGF3, interferon-stimulated gene factor 3; GAF, $\gamma$-activated factor; ISRE, interferon-stimulated response element; GAS, $\gamma$-activated sequence; ISG, interferon-stimulated gene; P, phosphate; IFNAR, IFN $\alpha$ receptor.

after IFN stimulation. These include members of the suppressors of cytokine signaling protein family (SOCS), in particular SOCS1. SOCS1 acts like a negative feedback loop for IFN-I signaling in a STAT1-dependent manner, by inhibiting JAK tyrosine kinase activity directly through its kinase inhibitory region (20).

\section{STAT2/IRF9: Phosphorylated STAT2 with IRF9}

Previously, we revealed that in the absence of STAT1, STAT2 homodimers interact with IRF9 that form the ISGF3-like complex STAT2/IRF9 and activate transcription of ISRE-containing genes in response to IFN $\alpha$ (21) (Figure 1). Under similar conditions, it was shown that an IRF9-STAT2 hybrid protein reinstates interferon-stimulated gene expression $(22,23)$. Multiple studies have subsequently provided evidence for the existence of a STAT1-independent IFN-I signaling pathway, where STAT2/
IRF9 substitutes ISGF3 function (24-27). STAT2 has been shown to heterodimerize with other STATs than STAT1. For example, in U266 cells STAT2 specifically interacted with STAT3 in an IFN-I-dependent manner in the presence of STAT1 (28). So far, however, no biochemical evidence exists that this complex together with IRF9 can reconstitute "ISGF3-like" functions in the absence of STAT1. Proof has been provided for an "ISGF3-like" role of STAT2-STAT6 heterodimers complexed with IRF9, but this complex seems to have a restricted role in B-cell specific IFN-I signaling (29).

Recently, more detailed insight was provided into the genomewide transcriptional regulation and the biological implications of STAT2/IRF9-dependent IFN $\alpha$ signaling as compared with interferon-stimulated gene factor 3 (ISGF3). In STAT2 overexpressing STAT1-deficient human and mouse cells, IFN $\alpha$-induced expression of typical ISGs correlated with the kinetics of STAT2 phosphorylation, and the presence of a STAT2/IRF9 complex. 
Results revealed that in the absence of STAT1, the STAT2/ IRF9 complex triggered expression of a similar subset of ISGs as ISGF3 (30) which is consistent with the observations of Lou et al. (31). It is also in agreement with the ability of STAT2 and IRF9 to move in and out of the nucleus as a complex (17). Among these commonly upregulated ISGs were known genes involved in antiviral response and within the promoters of all of these genes, we confirmed the presence of a classical ISGF3-binding ISRE (Figure 1). Interestingly, these genes exhibited different expression profiles: early and transient when driven by ISGF3 and delayed and prolonged expression when driven by STAT2/IRF9. This also correlated with the transient ISRE binding pattern of ISGF3 components as compared to the more prolonged binding of STAT2/IRF9 (Blaszczyk et al., manuscript in preparation). In this respect, Abdul-Sater et al. (32) showed that a prolonged activity of JAK1 and reduced levels of SOCS1 associated with the delayed kinetics of STAT2 activation (33). In human and mouse STAT1 KO cells overexpressing STAT2 cells (30) (data not shown) as well as in STAT1 KO BMM cells (32), reduced SOCS1 expression enables STAT2/IRF9-dependent and delayed expression of a subset of antiviral ISGs.

Our experiments also offered additional proof for the functional overlap between STAT2/IRF9 and ISGF3, by showing that the STAT2/IRF9 complex was able to trigger an antiviral response upon encephalomyocarditis virus and vesicular stomatitis Indiana virus (30). Thus, STAT2/IRF9 exhibits a biological function in the reconstitution of the antiviral response in cells lacking STAT1. In line with this, Yamauchi et al. recently showed in Huh-7.5 cells that IFN-I responses were only partially attenuated by knockout of STAT1 but completely by knockout of STAT2. Moreover, they observed that IFN-I inhibited hepatitis $\mathrm{C}$ virus (HCV) replication in a STAT2-dependent but STAT1independent manner (12).

Along the same lines, it was demonstrated that STAT2 plays a crucial role in a STAT1-independent protective mechanism against $L$. pneumophila and Denga virus infection. This highly suggests that DENV-mediated inactivation of STAT1 function alone is not sufficient to neutralize antiviral responses. More important, it is tempting to speculate that the STAT2/IRF9 pathway evolved as a backup response against pathogens that block STAT1 activity (32) [e.g., Paramyxovirus $(34,35)$ or Sendai virus (36)].

\section{GAF: Phosphorylated STAT1 Homodimers}

It has become clear that IFN-I is also able to regulate expression of a distinct set of genes through an additional STAT-based signaling cascade that depends on the formation of STAT1 homodimers (13-15) (Figure 1). Accordingly, binding of IFN-II to its tetrameric receptor, composed of the subunits IFNGR1 $(2 \times)$ and IFNGR2 $(2 \times)$, first leads to transphosphorylation and activation of JAK1 and JAK2. These JAKs then phosphorylate specific tyrosine residues of the IFN-II receptor, which serve as STAT1 docking sites, but not STAT2. Thus, IFN-II specifically triggers the tyrosine phosphorylation of STAT1 and homodimer formation. Subsequently, STAT1 homodimers (known as GAF) translocate into the nucleus to activate genes containing the GAS DNA element (13-15) (Figure 1). The NLS and NES within the DNA-binding domain of STAT1 are essential both for its nucleocytoplasmic shuttling and for regulating the amount of transcriptionally active GAF in the nucleus (37). As mentioned above, STAT1 homodimers are also formed after IFN-I treatment (Figure 1), however, because of direct competition with STAT1-STAT2 heterodimerization and ISGF3 complex formation, in much lower amounts (38). Therefore, in most cell types, the IFN-I-dependent GAF-activated pathway is less potent as compared to that dependent on IFN-II, which does not activate STAT1-STAT2 heterodimer formation. The GAS element is a palindromic sequence [consensus: $\operatorname{TTCN}_{(2-4)} \mathrm{GAA}$ ] that binds all STATs, except for STAT2. Thus, STAT1 homodimers bind to an element with canonical $N=3$ spacing (39). To date, many GAScontaining STAT1-target genes have been identified (40), including guanylate-binding protein (GBP), SOCS1, IRF1, and IRF8.

Like STAT1 and STAT2 phosphorylation in response to IFN-I, STAT1 phosphorylation upon IFN-II stimulation displays a rapid raise that drops in time and correlates with a transient ISG expression profile. Among the upregulated proteins is SOCS1, which culminates in the reduction of STAT1 phosphorylation after IFN-II stimulation (20).

\section{UNPHOSPHORYLATED STAT1 AND STAT2: U-ISGF3, U-STAT2/IRF9, AND U-STAT1}

\section{IFN Dependent}

Recent studies promote the model that U-STAT1 regulates expression of a subset of IFN-I-stimulated genes, including IFI27, BST2, OAS1, OAS2, OAS3, and STAT1 itself. Many of the encoded proteins display antiviral and immune regulatory activities $(2,3$, 10, 41-51). Supposedly, this occurred through the accumulation of newly synthesized STAT1 as part of a positive feedback loop after IFN stimulation (Figure 2) together with constantly replenished, but no longer phosphorylated, STAT proteins during process of nucleocytoplasmic shuttling (52). Moreover, prolonged exposure of cells to IFN- $\beta$ was proposed to induce the expression of unphosphorylated STAT2 (U-STAT2) and IRF9 which together with U-STAT1 form unphosporylated ISGF3 (U-ISGF3) (19) (Figure 2). Upon nuclear translocation, U-ISGF3 subsequently maintained the expression of a subset of the initially induced ISRE-containing ISGs, resulting in prolonged resistance to virus infection and DNA damage. Interestingly, the U-ISGF3dependent elongated expression of antiviral genes is possibly mediated by distinct ISREs (10).

A similar scenario was recently proposed for U-STAT2/IRF9 by Lou et al., in which abundance of U-STAT2 and IRF9 proteins mediated prolonged transcription of the $R I G-G$ gene independent of STAT2 phosphorylation, through the autocrine/paracrine action of secreted IFN $\alpha$ activated in all-trans retinoic acid-treated cells (Figure 2). This is in agreement with the ability of U-STAT2/ IRF9 to shuttle in and out of the nucleus (17). So far, it is not clear if in the absence of STAT1 this complex effects IFN-I-dependent expression of other ISRE-containing genes.

It was claimed that U-STAT1 does not activate gene expression as a homodimer $(10,53)$. However, Yao et al. recently observed in Mycobacterium tuberculosis (Mtb)-infected macrophages a rapid 


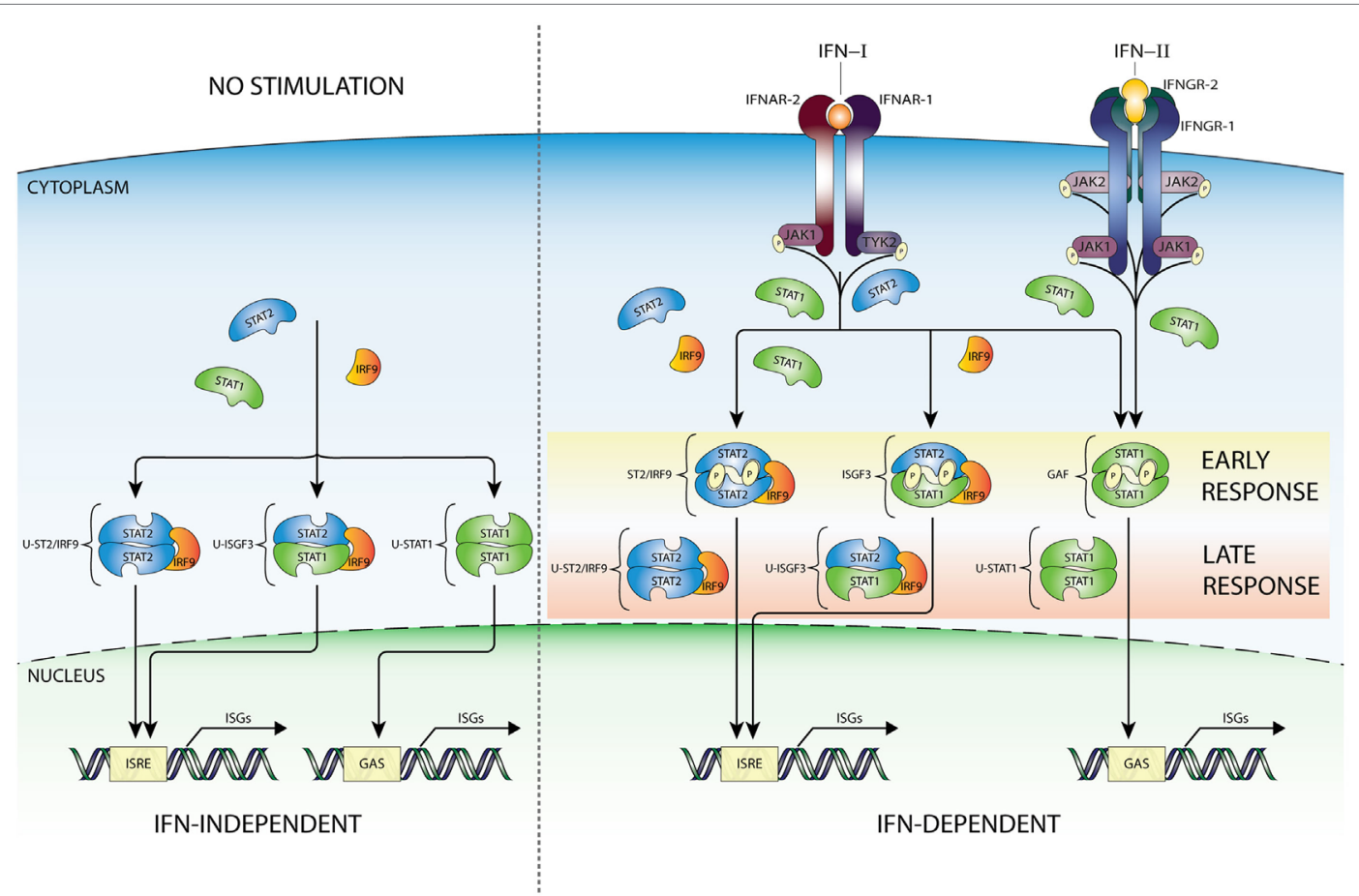

FIGURE 2 | IFN-dependent and -independent ISG transcription mediated by complexes comprised unphosphorylated STATs. In addition to the classical IFN signaling pathway based on complexes of phosphorylated STAT proteins (early response-details Figure 1), evidence exists for the involvement of unphosphorylated versions of ISGF3 (U-ISGF3), GAF (U-STAT1), and STAT2/IRF9 (U-STAT2/IRF9), in maintaining basal expression of certain ISGs in unstimulated cells (IFN independent). In addition, these unphosphorylated complexes are instrumental in sustaining expression of ISGs in response to long-term IFN-I or IFN-II stimulation (IFN dependent), when the level of phosphorylated STAT proteins is decreasing, but de novo synthesized unphosphorylated STAT proteins accumulate and can be incorporated in unphosphorylated transcription factors (late response). Abbreviations: IFN, interferon; STAT, signal transducer and activator of transcription; IRF, interferon regulatory factor; JAK, Janus kinase; TYK, tyrosine kinase; ISGF3, interferon-stimulated gene factor 3; GAF, $\gamma$-activated factor; ISRE, interferon-stimulated response element; GAS, $\gamma$-activated sequence; ISG, interferon-stimulated gene; P, phosphate; U, unphosphorylated form.

increase in phosphorylated STAT1, which quickly declined over a period of hours, but a continued increase of unphosphorylated U-STAT1 that persisted for several days. As such, U-STAT1 affected the expression of several immune-associated genes, and lowered sensitivity of macrophages to CD95-mediated apoptosis during $M t b$ infection (54). Likewise, Majoros et al. showed the importance of U-STAT1 in IFN-I-induced gene expression regulation and biological activity in mice expressing a Stat1Y701F mutant (19).

Therefore, in spite of virus-induced decline in IFN production, host cells can continue synthesizing antiviral proteins in a U-ISGF3-dependent manner, and possibly U-STAT2/IRF9 and U-STAT1, since even low concentrations of IFN-I can lead to increased expression of STAT1, STAT2, and IRF9 proteins (Figure 2). Thus, host cells can maintain at least some antiviral functions even after IFN synthesis decreases and phosphorylated signaling molecules are inactivated, by a supposedly tyrosine phosphorylation-independent mechanism. The expression of ISGF3 components is also highly increased in long-term IFNII-treated cells (11), providing the possibility for the formation of U-ISGF3 and U-GAF as well. Consequently, it is tempting to speculate that a similar U-ISGF3- or U-STAT1-dependent mechanism exists involved in long-term IFN-II-treated cells (Figure 2).

\section{IFN Independent}

Constitutive ISG expression in the absence of IFNs is known to be critical for cellular susceptibility to viral infection (2). In this respect, it has become clear that U-ISGF3, U-STAT1, and U-STAT2/ IRF9 can also mediate constitutive IFN-independent expression of ISGs to protect against viral infection (Figure 2). It has been reported, in different cell types, that U-STAT1 (52) and U-STAT2 (17) in combination with IRF9 are in constant motion between the cytoplasm and nucleus (37) and in the form of U-ISGF3 can be responsible for the basal activity of ISG promoters. For example, Wang et al. showed that in cell lines, three-dimensional (3D) cultured primary intestinal and liver organoids, and liver tissues under homeostatic conditions, endogenous STAT1, STAT2, and IRF9 could be observed in the nucleus. Indeed, under conditions without detectable IFNs, constitutive ISG expression was mediated by U-ISGF3 and correlated with genome-wide U-STAT1 binding to selective ISG promoters. This process effectively conferred resistance to HCV and HEV infections to host cells and was independent of IFN production and the upstream elements of IFN signaling (55). In conclusion, in host cells under homeostatic conditions U-ISGF3 was able to sustain constitutive ISG transcription and antiviral immunity. In STAT1 KO cells overexpressing STAT2 and IRF9, we observed that U-STAT2/IRF9 increases basal expression of several ISGs including IFI27, OAS2, OASL, and 
IFI44. As mentioned above, in untreated cells, U-STAT2/IRF9 is able to shuttle between the cytoplasm and the nucleus, with the potential to bind DNA and regulate expression of a selection of ISGs. Therefore, we propose a comparable set-up in the absence of STAT1, that abundant U-STAT2 and IRF9 proteins can form a complex and drive ISG expression independent of IFN treatment [(30); Nowicka et al. data not shown].

Finally, basal DNA-binding of U-STAT1 is connected to its nuclear localization as well as the constitutive expression of some targets $(56,57)$. For example, U-STAT1 complexes with IRF1 at the LMP2 and TAP2 promoters and maintains its constitutive expression (56).

Thus, these observations suggest that both IFN-dependent and IFN-independent antiviral mechanisms are present simultaneously and act in a cooperative fashion (Figures $\mathbf{1}$ and 2).

\section{IRF1 IN ISG TRANSCRIPTIONAL REGULATION}

Interferon regulatory factors are a family of 9 transcription factors (IRF1-9) which display a diversity in functions, including immune cell development cell cycle regulation, apoptosis, host defense, and oncogenesis $(58,59)$. IRFs all contain a conserved DNA-binding domain and IRF association domain. The DNAbinding domain consists of a five-tryptophan repeat that is located at the amino terminus and binds to a specific GAAA motif (IRF element: IRE), present within the promoters of IFN $\alpha$ and IFN $\beta$ genes as well as in ISGs (ISRE core). The IRE is a shorter version of the ISRE, not recognized by ISGF3. IRF1 and IRF9 are induced by both IFN-I and -II and viral infection $(24,58,59)$. Like IRF9, IRF1 has also been shown to regulate transcription of ISGs in response to different types of IFNs. Nuclear localization of the IRF1 protein is regulated by an active NLS located immediately C-terminal to the DNA-binding domain (60). IFN-I and IFN-II induced transcriptional regulation of the IRF1 gene identified a single GAS element (TTTCCCCGAAA) and no ISRE in its proximal promoter (40), shown to bind GAF (Figure 3). As mentioned above, STAT1 homodimers are also formed after IFN-I treatment, however, usually in much lower amounts. Therefore, in most cell types IRF1 is more potently induced by IFN-II as compared to IFN-I (38). In mouse IRF9 KO embryonic fibroblasts (61) as well as in human IRF9 deficient U2A cells (62), IFN-I and IFN-II induction of IRF1 is not

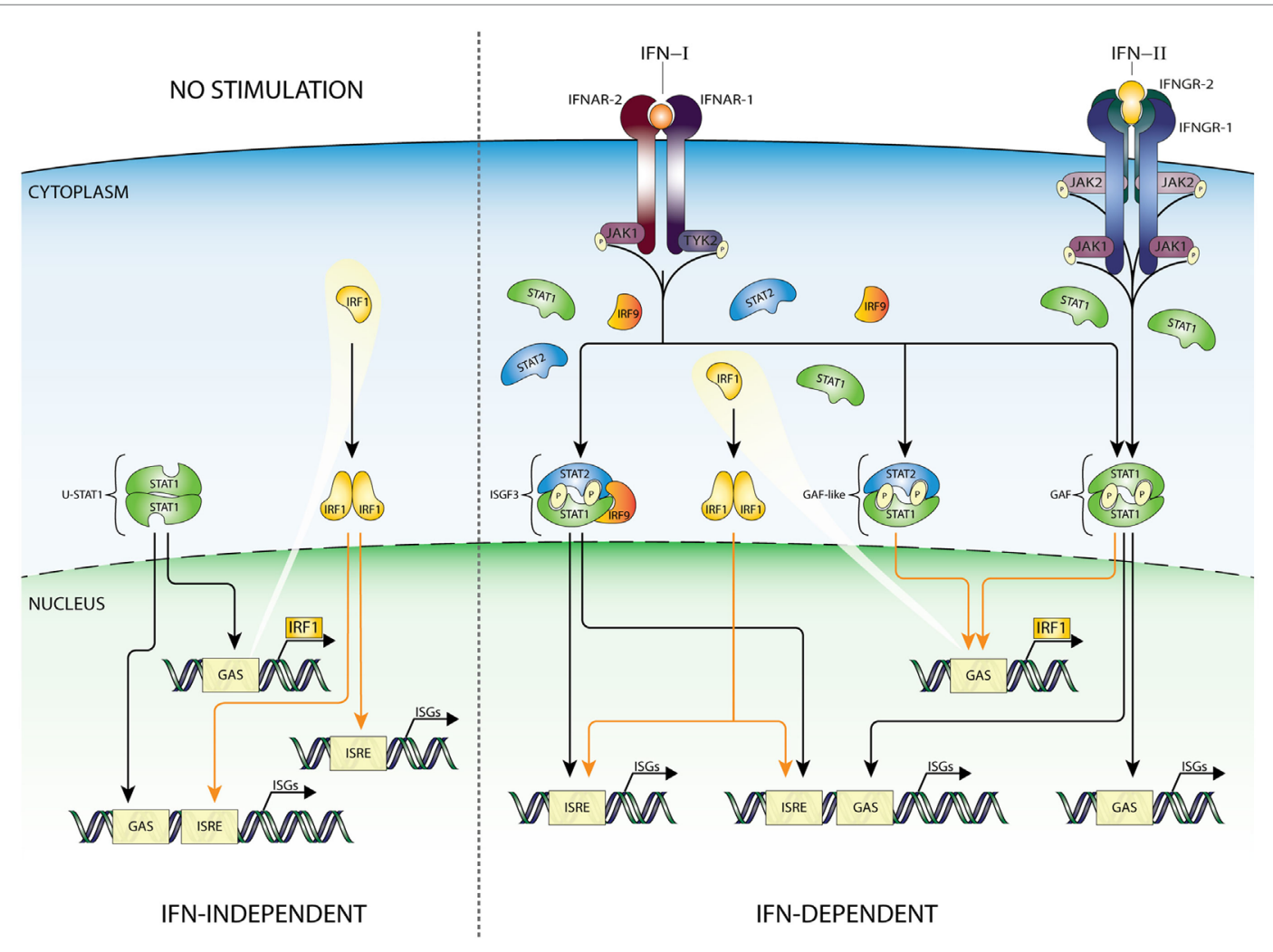

FIGURE 3 | IFN-dependent and -independent ISG transcription mediated by IRF1. According to the current state of knowledge, expression of IRF1 is driven by homodimers of STAT1 (GAF), as well as STAT1/STAT2 heterodimers, recognizing a single GAS sequence in the IRF1 promoter. Recent studies confirmed that basal expression of IRF1 can be detected in many cell types under basal conditions. Moreover, binding of IRF1 to ISRE-containing genes can play a role in maintaining constitutive expression of certain group of ISGs (left panel). After IFN-I or IFN-II stimulation GAF and GAF-like (STAT1/STAT2 heterodimers) complexes are rapidly formed and translocated to the nucleus to initiate IRF1 expression. Subsequently, IRF1 as an additional abundant transcription factor can collaborate with ISGF3 and GAF complexes stimulating ISRE-containing genes, thus appearing as an important link between IFN-I and IFN-II responses. Abbreviations: IFN, interferon; STAT, signal transducer and activator of transcription; IRF, interferon regulatory factor; JAK, Janus kinase; TYK, tyrosine kinase; ISGF3, interferon-stimulated gene factor 3; GAF, $\gamma$-activated factor; ISRE, interferon-stimulated response element; GAS, $\gamma$-activated sequence; ISG, interferon-stimulated gene; P, phosphate. 
significantly different from Wt cells. On the other hand, IFN-I upregulation of IRF1 in U3C-STAT2 cells is severely hampered, consistent with the notion that GAF is a critical mediator and not ISGF3 or STAT2/IRF9 (10, 40, 63). Interestingly, in U2A cells, IFN-I-induced phoshoSTAT1-STAT2 heterodimers (pSTAT1STAT2) were also shown to bind the IRF1 GAS element and to act as a potent transcriptional activator of the $I R F 1$ gene $(28,62$, 64-67) (Figure 3).

As described above, IRF9 complexes with STATs and redirects them to ISRE-containing ISGs. By contrast, IRF1 regulates ISG transcription through homo- or heterodimerization and binding to IRE or ISRE sequences. Thus, as a STAT1-target gene, IRF1 participates in secondary IFN-I and -II responses by activating transcription of ISRE-containing genes (Figure 3). Microarray analysis of IRF1 overexpressing Huh-7 and STAT1-/- fibroblasts identified many IRF1-target genes, including known antiviral ISGs as well as STAT1, STAT2, and IRF9 (48). In a different study, it became clear that IRF1 restricts HEV replication by directly regulating expression of STAT1, resulting in increased levels of total and phosphorylated STAT1 protein and subsequently a panel of downstream antiviral STAT1-target genes (68). Furthermore, the antiviral activity of IRF1 was dependent on the JAK-STAT signaling pathway, but independent of IFN production. Together, this points to a functional overlap between IRF1 and ISGF3 and a role of IRF1 in regulating expression of ISGF3 components.

By generating mice deficient for both IRF9 and IRF1 alleles, Kimura et al. studied the functional interaction of IRF9 and IRF1 in IFN-I and IFN-II-induced gene regulation. For example, it was shown that IFN induction of the ISRE-containing genes $O A S$ and $P K R$ is primarily regulated by IRF9, in the context of ISGF3; i.e., IRF1 cannot compensate for the loss of IRF9 (61). Interestingly, induction of GBP by IFN-I required both IRF1 and IRF9, consistent inducible genes $(69,70)$. In contrast to IFN-I, IFN-II induction of GBP mRNA was largely dependent on IRF1. Thus, the GBP promoter differentially utilizes IRF9 and IRF1, depending on the type of IFN used to stimulate the cells.

Collectively, this highly suggested that IRF1 and ISGF3 are not functionally redundant but complement each other and collaborate to ensure the induction of the full range of overlapping target genes which respond to IFN-I and IFN-II (Figure 3).

Collaborations between IRF1 and STAT1 in transcriptional regulation of ISRE and GAS-containing ISGs have also been described (Figure 3). For example, the IFN $\gamma$-induced expression of CIITA, GBP1, and gp19 was shown to depend on both STAT1 and IRF1 (71-73). Moreover, an independent study of 128 transcription factors in IFN $\gamma$ treated K562 cells unraveled that STAT1-IRF1 co-binding is a general phenomenon (74). It is tempting to speculate that a similar mechanism of STAT1IRF1 co-binding plays a role in IFN-I-mediated transcriptional responses (Figure 3).

In an IFN-independent manner, basal expression of IRF1 can be detected in many cell types. Correspondingly, constitutive binding of IRF1 was shown to regulate constitutive ISG expression, either alone or in combination with STAT1 $(56,75)$ (Figure 3).

\section{GERMLINE MUTATIONS OF STAT1 AND STAT2 AND THE PRESERVATION OF IFN RESPONSES}

\section{STAT1}

In 2001, Dupuis et al. described three patients carrying a heterozygous germline L706S STAT1 mutation, which lead to an impaired ability to phosphorylate and translocate mutated STAT1 protein to the nucleus in response to IFN stimulation. Translocation of STAT2 according to immunofluorescence was not changed and expression of the ISFG3 target gene-MXA upon IFN $\alpha$ treatment was not reduced. These patients were susceptible to mycobacterial but not viral infections (76). Similar results were shown for patients with an autosomal dominant M654K STAT1 mutation with disrupted IFN-II response, but mildly influenced IFN-I response (77). Likewise, three other patients with identified STAT1 alleles (E320Q, Q463H, and L706S) that profoundly diminished IFN-II mediate ISG expression and responses to mycobacteria did not affect IFN-I-activated transcription and antiviral responses. Thus, indirect evidence exists that in patients with diminished STAT1 levels and/or function cellular mechanisms are present that allow preservation of intact antiviral responses (78).

On the other hand, a report about two unrelated infants with homozygous STAT1 deleterious mutations revealed for the first time that complete lack of STAT1 production abolishes not only IFN $\gamma$ signaling but also IFN $\alpha / \beta$ response in vivo, which resulted in susceptibility and death after viral infection (79). This observation correlated with studies conducted on mouse STAT1-/- fibroblasts transfected with mutated L706S STAT1 alleles, showing disabled GAF and ISGF3 formation and disrupted translocation of the STAT2 protein to the nucleus in response to IFN. This was explained by possible sequestration of ISGF3 components by mutated STAT1 protein and its retention in the cytoplasm (76). It is also in agreement with later studies describing the phenomenon of mice with a homozygous mutation Y701F STAT1, which were more susceptible to infection than STAT1-null mice and were not able to develop a similar type of STAT2/IRF9-mediated response to IFN as seen in STAT1-null mice (19).

Apparently, not only loss-of-function STAT1 mutations are responsible for changed immunological responses. Unbalanced STAT1 hyperactivity in case of gain-of-function mutations also leads to the development of chronic mucocutaneous candidiasis or autoimmunological diseases (80).

\section{STAT2}

Reported cases of STAT2 deficiency are always connected with a background of viral infections. Patients carrying STAT2 mutations are in general not able to induce full ISGF3-dependent IFN responses. The GAF-dependent IFN-I and IFN-II responses remain mostly unaffected. Moens et al. described a patient with a heterozygous STAT2 mutation who suffered from severe viral infections since infancy. Patient's fibroblasts do not express STAT2 in full length or truncated form and after stimulation with IFN $\alpha$ ISRE-containing genes (MX1, ISG15, and OAS1 were checked) were not induced. After transfection with wtSTAT2, cells restored IFN-inducible STAT2 phosphorylation and ISG expression (81). 
In another case study of siblings with STAT2 deficiency, the authors used whole-genome transcriptional profiling of patient's fibroblasts with or without stimulation of IFN $\alpha$. Only $\sim 10 \%$ of upregulated genes in control cells were also upregulated in patient's fibroblast. The residual upregulated ISGs were largely overlapping with these from control but expressed at lower level, except IRF1. Interestingly, promoter transcription factor binding site prediction revealed enrichment of GAF and/or IRF1 binding elements in the promoters of expressed ISGs (82). These observations suggest that type I IFN signaling (through ISGF3) is not completely essential for host defense against viral infections.

Together, from STAT1 and STAT2 germ-line mutation studies, it can be concluded that well-balanced and strictly controlled IFN-I and/or IFN-II-mediated immune responses are necessary but not sufficient to fight with infection. Moreover, potential backup systems, including STAT2/IRF9 or IRF1, could be activated under conditions where STAT1 or STAT2 are not fully active.

\section{GENOME-WIDE BINDING OF STAT1, STAT2, IRF9, AND IRF1 AND REGULATION OF ISRE AND GAS-DEPENDENT TRANSCRIPTION}

Most of the knowledge about the DNA responsive elements involved in IFN-I and IFN-II signaling dates from early experiments that focused on individual genes and their role in the antiviral response (83). Accordingly, the ISRE was shown to exist in proximal ISG promoters as a single element or in multiple copies, in either orientation with (minor) consensus sequence variations (AGTTTCN2TTTCN; Table 1). Functional analysis of a selection of IFN-I-inducible genes $(84,85)$, as exemplified in Table 1, has revealed that ISRE is essential for IFN induction.

Studies of the GBP gene, which is inducible by both IFN $\gamma$ and IFN $\alpha / \beta$, has led to the identification of the IFN $\gamma$ activation site (GAS, consensus sequence: TTCCNGGAA (40); Table 1), in addition to an $\operatorname{ISRE}(104,105)$. Subsequently, GAS was shown to be involved in the IFN-II-mediated transcriptional activation of several other genes and predominantly localized in their proximal promoters (Table 1). In the GBP gene, an ISRE was also found juxtaposed to the GAS, and both elements clearly contributed to both IFN-I and IFN-II responses $(105,106)$. Additional genes, stimulating cooperation of ISGF3 and GAF on ISRE and GAS composites have since been identified $(40,107)$ (Table 1). Cooperation between STAT1 and IRF1 in the IFN $\gamma$-regulation of GAS and ISRE-containing ISGs, has also been suggested $(73,107)$. In addition, binding modes exist in which multiple GAF complexes can be recruited to adjacent GAS sites $(13,108)$. Similar cooperative DNA-binding has been described for ISGF3 (109).

\section{IFN Dependent}

More recent genome-wide binding approaches (using ChIP-chip or ChIP-seq), monitoring binding of STAT1, STAT2, and IRF1 in response to IFN-I or IFN-II, confirmed this genomic organization and identified many known and novel target genes with varying binding modes to individual or combined ISRE and GAS sites
(83). For example, Hartman et al. used chromatin immunoprecipitation and DNA microarray analysis to identify STAT1 and STAT2-binding regions on chromosome 22 in IFN-treated cells (110). Markedly, non-conserved STAT1 occupancy was detected at GAS sites as well as novel STAT1 binding sites upon IFN $\alpha$ induction, not observed in IFN $\gamma$-treated cells. As expected, a large number of these sites correlated with STAT2 binding. However, others appeared STAT2 independent. Moreover, novel STAT2binding sites could be identified, without STAT1, suggesting that under different activation conditions various mechanisms direct STAT1 and STAT2 binding to their targets.

Closer inspection of the publicly accessible dataset (111) featuring STAT1, STAT2, and IRF1 ChIP-seq experiments performed on chromatin extracted from K562 cells treated with IFN $\alpha$ or IFN $\gamma$ for $30 \mathrm{~min}$ or $6 \mathrm{~h}$ (www.encodeproject.org), could recognize the binding to typical ISRE or GAS-containing genes of known and novel origin (Table 1; Figure 4). In addition, it revealed the existence of a large group of IFN-I and IFN-II activated genes that use ISRE and GAS composite sites (see Table 1). These observations were in line with our previous generated ChIP-seq dataset that examined binding of STAT1, STAT2, and IRF9 in IFN $\alpha$-treated and untreated 2fTGH cells (Figure 4; Bluyssen et al., manuscript in preparation). The ISRE-only genes, ISG15, MX1, OAS3, and IFIT3 clearly only bind ISGF3 in response to IFN-I (as represented by co-binding of STAT1, STAT2, and IRF9) as well as IRF1 after IFN-I and IFN-II stimulation (Figure 4), whereas the GAS-only genes (i.e., IRF1, SOCS3, and ICAM1) strictly bind GAF and/or pSTAT1-STAT2 under these conditions (Figure 4). By contrast, the ISRE and GAS composite-containing genes, exemplified by IFI35, DTX3L, TRIM69, AIM2, BST2, SOCS1, STAT1, STAT2, and IRF9, display a binding pattern of STAT1, STAT2, IRF9, and IRF1 that points to a mechanism of co-binding of ISGF3, IRF1, or GAF complexes in response to IFN-I or IFN-II (Figure 4). The presence of these different ISG sub-groups is in agreement with previous data $(107,112)$. Hassan et al. further highlighted the importance of STAT1 and IRF1 cooperation by detailed studies of ISG-rich chromosomal segments (containing 10\% of all known ISGs) in HeLa cells. Under these conditions, most ISG-rich loci responded to IFN $\gamma$, with only $\sim 20 \%$ of ISGs being unresponsive. IRF1 binding sites were detected twice as often as STAT1 sites, with isolated IRF1 binding as a consequence. On the other hand, most STAT1 binding occurred at or near to IRF1 sites (dual binding), which was closely linked to ISG responsiveness (75).

Presently it is not clear how these ISRE and GAS compositecontaining genes are represented within the full spectrum of IFN-I and IFN-II induced ISGs and how they contribute to overall IFN-mediated antiviral activities.

At the same time, these genome-wide binding approaches concluded that STAT1, STAT2, and IRF9 bind not only at promoter proximal but also distal ISRE and/or GAS sites suggesting a role of distant enhancers in remote gene regulation (83). Indeed, it was shown before that IFN $\gamma$ induces long-range interactions between STAT1-bound enhancers and target promoters (75, 113-115). Moreover, several studies reported the functionality of typical enhancers, involving long-range interactions and IFN-induced recruitment of STAT1 to distal regulatory regions. For example, Li et al. showed for the IFITM1, 2, and 3 gene 
TABLE 1 | ISRE and GAS sequences of known ISGs.

\begin{tabular}{|c|c|c|c|c|c|c|}
\hline Type TFBS & Gene name & $\begin{array}{l}\text { ISRE consensus sequence } \\
\text { AG-TTT-CNN-TTT-CN }\end{array}$ & Linker $^{\mathrm{a}}$ & $\begin{array}{l}\text { GAS consensus sequence } \\
\text { TTC-CNG-GAA }\end{array}$ & Location in relation to TSS & Reference \\
\hline \multirow[t]{11}{*}{ ISRE } & $\mathrm{MX} 1$ & $\begin{array}{l}\text { AG-TाT-CGG-TT-CA } \\
\text { GG-TT-CG-TT-CT } \\
\text { AG-TाT-CA-TाT-CT }\end{array}$ & & $\begin{array}{l}- \\
- \\
-\end{array}$ & $\begin{array}{l}\text { Proximal } \\
\text { Intragenic }\end{array}$ & (86) \\
\hline & ISG15 & $\begin{array}{l}\text { AG-TाT-CGG-TाT-CC } \\
\text { GG-TाT-CCC-TाT-CC }\end{array}$ & & - & Proximal & $(87)$ \\
\hline & IFIT1 & AG-TाT-CAC-TाT-CC & & - & Proximal & (85) \\
\hline & IFIT2 & AG-TाT-CAC-TाT-CC & & - & Proximal & (85) \\
\hline & IFIT3 & 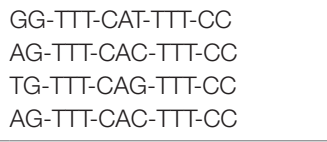 & & $\begin{array}{l}- \\
- \\
- \\
-\end{array}$ & $\begin{array}{l}\text { Proximal } \\
\text { Intragenic }\end{array}$ & $(87)$ \\
\hline & USP18 & $\begin{array}{l}\text { AG-TाT-CGC-TाT-CC } \\
\text { GC-TIT-CGT-TIT-CC }\end{array}$ & & - & Proximal & $(87)$ \\
\hline & OAS1 & GG-TाT-CG-TाT-CC & & - & Proximal & (88) \\
\hline & OAS2 & AG-TाT-CAG-TाT-CC & & - & Proximal & (89) \\
\hline & OAS3 & $\begin{array}{l}\text { GG-TIT-CGT-TIT-CC } \\
\text { GC-TIT-CAG-TIT-CG }\end{array}$ & & - & Proximal & (90) \\
\hline & OASL & AG-TाT-CGA-TT-CT & & - & Proximal & (91) \\
\hline & ISG20 & TG-TाT-CAG-TाT-CT & & & Proximal & $(92)$ \\
\hline \multirow[t]{4}{*}{ GAS } & IRF8 & $\begin{array}{l}- \\
-\end{array}$ & & $\begin{array}{l}\text { TाT-TCG-GAAA } \\
\text { TTC-GAA-GAA }\end{array}$ & Proximal & (88) \\
\hline & SOCS3 & - & & TTC-CTG-GAA & Proximal & (93) \\
\hline & ICAM1 & - & & TाTC-CGG-GAAA & Proximal & (94) \\
\hline & IRF1 & $\begin{array}{l}- \\
- \\
-\end{array}$ & & $\begin{array}{l}\text { TाTC-CCC-GAAA } \\
\text { TाC-TTA-TAAA } \\
\text {-TTC-CTG-GAAA }\end{array}$ & $\begin{array}{l}\text { Proximal } \\
\text { Distal }-6 \mathrm{~kb}\end{array}$ & $\begin{array}{c}(95,96) \\
\text { b }\end{array}$ \\
\hline \multirow[t]{12}{*}{ ISRE/GAS } & STAT1 & $\begin{array}{l}\text { AG-TाT-CGC-TाT-CC } \\
\text { CT-TाT-CGG-Tा-CC }\end{array}$ & $32 \mathrm{nt}$ & TाTC-CCC-GAAA & $\begin{array}{l}\text { Proximal } \\
\text { Distal }-5.5 \mathrm{~kb}\end{array}$ & (97) \\
\hline & STAT2 & $\begin{array}{l}\text { AG-TIT-CGG-TT-CC } \\
\text { CA-TIT-CTC-TIT-AT }\end{array}$ & $15 \mathrm{nt}$ & TाIT-CTC-GAA & $\begin{array}{l}\text { Proximal } \\
\text { Intronic }\end{array}$ & (98) \\
\hline & IRF9 & AG-TाT-CAG-TT-CT & $16 \mathrm{nt}$ & TाT-CCA-GAAA & Proximal & $(87)$ \\
\hline & IFITM1 & AG-TIT-CTA-TIT-CC & $16 \mathrm{nt}$ & TाTC-TCA-GAA & Proximal & $(87,99)$ \\
\hline & BST2 & AG-TाT-CAG-TाT-CC & Overlap & TाT-CCA-GAAA & Proximal & $(100)$ \\
\hline & TAP1 & GA-TIT-CGC-TIT-CC & Overlap & ПTТ-CCC-TAAA & Proximal & $(101)$ \\
\hline & SOCS1 & $\begin{array}{l}\text { GG-TIT-CAC-TIT-CA } \\
\text { AC-TIT-CAG-TIT-CT }\end{array}$ & $\begin{array}{l}1 \mathrm{nt} \\
\text { Overlap }\end{array}$ & TाTC-CAA-GAAA & Distal -55 kb & (75) \\
\hline & IFI35 & AC-TाT-CA-TाT-CC & Overlap & TाTC-CGT-GAAA & Proximal & $(102)$ \\
\hline & HLA-G & AG-TIT-CAC-TाT-CC & Overlap & गाC-GA-GAA & Proximal & $(103)$ \\
\hline & ZC3HAV1 & GC-TाT-TAG-TाT-CT & $95 \mathrm{nt}$ & -TTC-CCG-GAAA & Proximal & (91) \\
\hline & $\mathrm{AlM}^{\mathrm{b}}$ & AC-TाT-CGC-TT-GG & $149 \mathrm{nt}$ & गाC-TGG-GAAA & Proximal & \\
\hline & TRIM69º & GG-TाT-CTC-TाT-CT & $14 \mathrm{nt}$ & TाTC-CGA-GAAA & Distal $-7.5 \mathrm{~kb}$ & \\
\hline
\end{tabular}

${ }^{a}$ Distance between elements if both are present.

bUnpublished data.

ISG, interferon-stimulated gene; GAS, $\gamma$-activated sequence.

cluster that STAT1 binding (and possibly that of STAT2) to the E2-3 enhancer, which was located $>35 \mathrm{~kb}$ away from the IFITM gene cluster, was indispensable for its IFN-responsive enhancer activity (116). It is quite interesting that the promoters of IFITM genes already contain the binding sites for STAT1 or STAT2 while there still exists an enhancer mediating IFN/STAT signaling. A reasonable explanation might be that the distal enhancer could fine-tune the expression of these important genes and thus add robust and plastic response to virus invasion and IFN stimulation. Likewise, Yuasa and Hijikata (117) identified a novel distal 


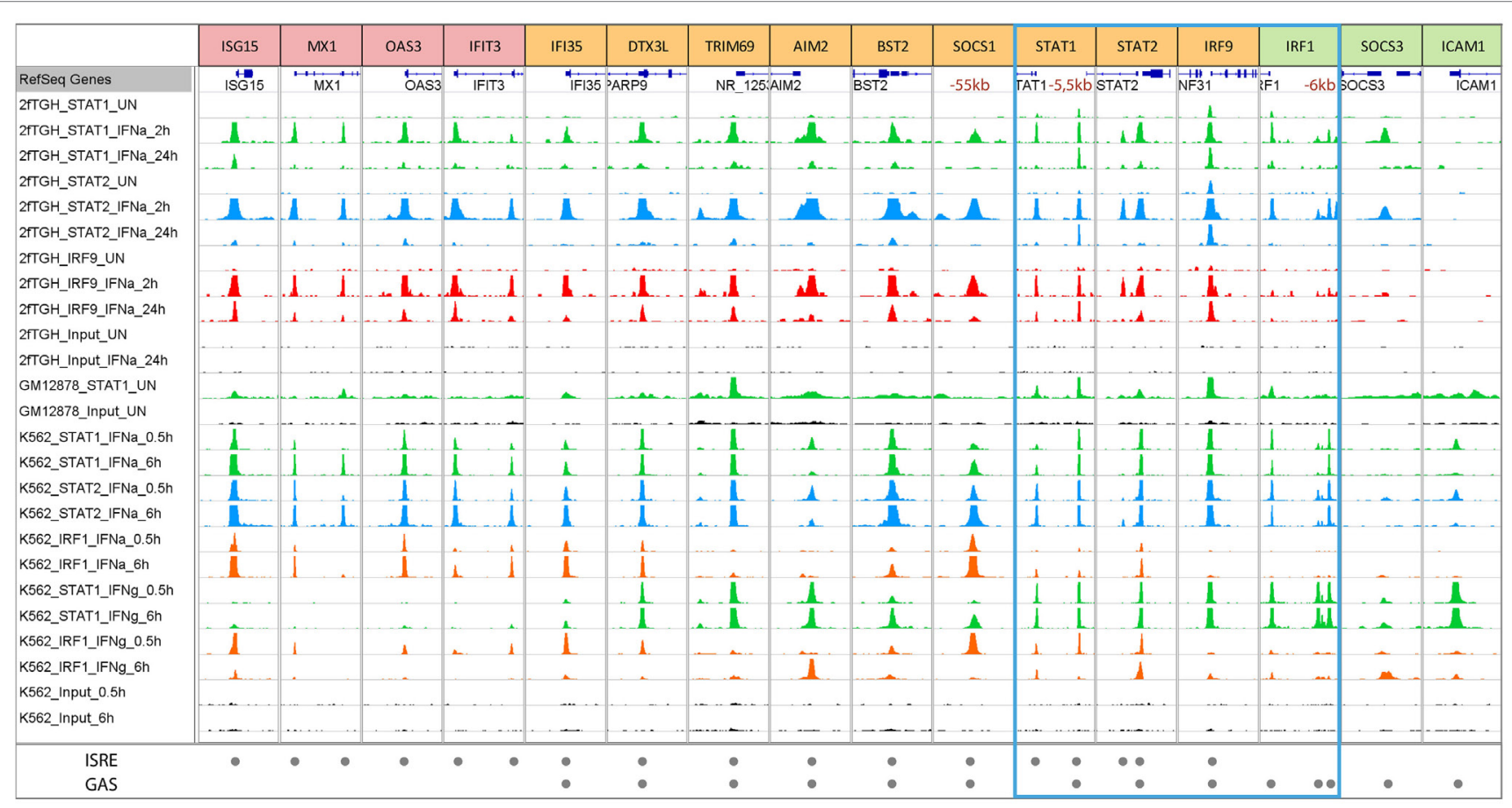

FIGURE 4 | Representative modes of STAT1, STAT2, IRF9, and IRF1 binding to ISRE and GAS-containing ISGs in GM12878, K562, and 2fTGH cells treated with or without IFN-I or IFN-II. To further study genome-wide binding of STAT1, STAT2, IRF9, and IRF1, a ChIP-seq dataset from K562 cells treated with IFN $\alpha$ or IFN $\gamma$ for 0.5 or $6 \mathrm{~h}$ and GM12878 cells used as a control were retrieved from the ENCODE database (GSE31477) and compared to our recently dataset from 2fTGH cells treated with or without IFNo for 2 and $24 \mathrm{~h}$ (unpublished data). Antibodies used in given experiments are indicated in the left panel and by color of the track-STAT1green, STAT2-blue, IRF9-red, and IRF1 - orange. Tracks derived from input sequencing are indicated in black. The bottom panel indicates ISRE or/and GAS sequences located under the peaks (see also Table 1). According to DNA motifs, ISGs can be divided into three groups: only ISRE-containing genes (examples are presented in red boxes -ISG15, MX1, OAS3, and IFIT3), only GAS-containing genes (examples in green boxes -IRF1, SOCS3, and ICAM1), and genes with both sequences (examples in yellow boxes-IFI35, DTX3L, TRIM69, AIM2, BST2, SOCS1, STAT1, STAT2, and IRF9). The blue frame marks components of ISGF3, GAF, and IRF1. Each group of genes displays differences in binding after IFN stimulation. ISRE-only genes show strong binding of components of ISGF3 (STAT1, STAT2, and IRF9) after IFN $\alpha$, as well as IRF1 after IFN $\alpha$ and IFN $\gamma$, but no binding of STAT1 after IFN $\gamma$ treatment. On the other hand, GAS-only genes demonstrate strong binding of STAT1 and STAT2 (components of GAF and GAF-like complexes) after IFN $\gamma$ and IFN $\alpha$ treatment. The third group consists of ISRE + GAS composite-site containing ISGs, which display binding of all the components in response to both types of IFNs pointing to the possible cooperation and mechanism of co-binding of ISGF3, GAF and IRF1 for optimal gene expression. Abbreviations: IFN, interferon; STAT, signal transducer and activator of transcription; IRF, interferon regulatory factor; ISGF3, interferon-stimulated gene factor 3; GAF, $\gamma$-activated factor; ISRE, interferon-stimulated response element; GAS, $\gamma$-activated sequence; ISG, interferon-stimulated gene.

regulatory element (5.5URR) positioned 5.5-kb upstream of the mouse STAT1 gene (117). The 5.5URR is evolutionary highly conserved in its upstream localization of the STAT1 gene and presence of a combined ISRE and a GAS site (118). By association of STAT1 complexes, namely ISGF3 and GAF, this ISRE and GAS composite was shown to physically associate with the STAT1 core promoter and proposed to mediate the autoregulation of the STAT1 gene and its prolonged expression in response to IFN-I and IFN-II (117). Examination of the above described ChIPseq datasets (K562 and 2fTGH), identified the presence of the 5.5URR enhancer also in the human STAT1 gene with apparent co-binding of ISGF3, GAF, and/or IRF1 in response to both types of IFN (Table 1; Figure 4).

ChIP-chip and ChIP-seq studies also showed that IRF1 binds many distal ISRE-containing enhancers $(75,83,119-121)$. For example, IRF1 binds to remote enhancers of the CIITA locus that form a 3D interconnected loop with the promoter (115). Similarly, functionality of putative distal enhancers at the SOCS1 locus were confirmed, combined with a co-binding role of IRF1 and STAT1 (75). In particular, the distal enhancer at $-55 \mathrm{~kb}$ from the transcriptional start site of the human SOCS1 gene was indispensable for its IFN-responsive enhancer activity. According to the above described ChIP-seq datasets (K562 and 2fTGH), a comparable binding pattern of STAT1 and IRF1 could be observed to this distal ISRE and GAS-containing enhancer of SOCS1 (Table 1; Figure 4).

The function of the majority of distal ISGF3, GAF, and IRF1 binding sites remains largely unknown. Nevertheless, it predicts the presence of a common regulatory mechanism of ISG transcriptional regulation.

\section{IFN Independent}

IFN independent, basal expression of some ISGs has recently been linked to the nuclear localization of U-ISGF3 and U-GAF $(56,57)$. Accordingly, Wang et al. extracted genome-wide STAT1 ChIP-seq data (GSE31477) from the ENCODE ChIP-seq 
Experiment Matrix database and Gene Expression Omnibus (111) and observed that even in the absence of IFN stimulation STAT1 displayed specific binding to promoter regions of an extensive group of ISGs (186 of 350 ISGs analyzed), including IRF1, IRF9, STAT1, and ISG15 (55). As becomes clear from Figure 4, after further examination of this dataset (GSE31477) (and to a lesser extent from our 2fTGH dataset), a selection of ISRE-only (ISG15 and MX1), GAS-only (IRF1 and ICAM1), and ISRE GAS composite genes (IFI35, TRIM69, AIM2, STAT1, STAT2, and IRF9) display binding of STAT1 under basal conditions. This is in agreement with a predicted role of U-ISGF3 or U-STAT1 in IFN-independent ISG expression $(55,75)$. At the same time, these observations coincide with the fact that this is not a general phenomenon for all ISGs, but apparently concerns a selected group (55).

Interferon regulatory factor 1 is also expressed at low levels in many unstimulated cell types, including HeLa cells (115), and it collaborates with U-STAT1 in maintaining low basal expression of for example LMP2 (56). Likewise, constitutive IRF1 binding facilitates constitutive PSMB9 and TAP2 expression $(56,122)$. Using ChIP-ChIP, Hassan et al. detected a number of U-STAT1 and IRF1 binding sites in untreated cells, accounting for 2.2 and $14.3 \%$ of induced sites, respectively (75). In addition, the basal expression of all of these genes was confirmed by microarray and/or RT-PCR (75). These data accord with another ChIP-chip analysis of STAT1 binding in HeLa cells treated for $30 \mathrm{~min}$ with IFN $\gamma$, observing $6.5 \%$ of IFN $\gamma$-induced STAT1 sites being occupied in unstimulated cells (123). In addition, basal TF binding loci were present in paralogous gene clusters predicting a link between gene duplication and high affinity binding site conservation (e.g., PSMB8 and PSMB9, GBP2 and GBP3, and IFIT1, IFIT2, and IFIT3). Moreover, the majority of the IRF1 basally occupied sites possessed ISRE motifs (75).

In a recent ChIP-chip study by Testoni et al., using anti-STAT2 and anti-phosphoSTAT2 antibodies, U-STAT2 was shown to be present in the nucleus of untreated cells and already bound to $62 \%$ of its target promoters, including many "classical" ISGs (87). This implies that under basal conditions nuclear U-STAT2/IRF9 binds genome-wide to ISG ISREs, including those encoding ISGF3 components, and keeping their expression at a low level.

Thus, increasing evidence supports the notion that basal genome-wide binding of U-ISGF3, U-STAT2/IRF9, U-GAF, and IRF1 is physiologically relevant.

\section{ISRE AND GAS-DEPENDENT REGULATION OF THE STAT1, STAT2, IRF9, AND IRF1 GENES: A MODEL OF POSITIVE FEEDBACK REGULATION}

\section{IRF1}

As mentioned above, transcriptional regulation of the IRF1 gene in response to IFN-I and IFN-II depends on a single GAS element. According to the above-described ChIP-seq datasets (K562 and 2fTGH), clear binding of STAT1 and STAT2, but no IRF9 or IRF1, was observed at the same position in the proximal $I R F 1$ promoter (Figure 4; left peak) in a time-dependent manner.
This corresponds with the presence of a single GAS element and no ISRE in the IRF1 proximal promoter (Table 1) and with previous studies $(62,67)$, in which a heterodimer of STAT1 and STAT2 (without IRF9) was shown to bind to this site and regulate expression of the IRF1 gene (62) in response to IFN-I. Moreover, binding of STAT1 (but not IRF1) to this proximal GAS in the IRF1 promoter in response to IFN-II (Figure 4; K562 cells only), is in line with the functional role of a STAT1 homodimer-mediated transcription (95). Closer examination, identified a similar binding pattern of STAT1, STAT2, IRF9, and IRF1 in response to IFN-I and IFN-II in the two different cell lines on more distal sites in the IRF1 promoter (Figure 4: right peaks; Table 1: first and second distal), which likewise corresponded with the presence of putative GAS sites in the sequences under the peak (Table 1). The functionality of these sites in relation to IRF1 expression is currently not known. Basal binding of U-STAT1 to the IRF1 proximal promoter, as observed in the GSE31477 dataset (Figure 4), correlates with IFN-independent expression of IRF1 (Figure 3) (75).

\section{STAT1, STAT2, and IRF9}

In many cell types, expression of STAT1, STAT2, and IRF9 is increased in response to IFN-I and IFN-II, being detectable even several days after treatment $(10-12,107)$. This IFN-dependent expression relies on the presence of ISRE and GAS-containing regulatory sequences.

In relation to the STAT1 gene, several elements have shown to be involved in its transcriptional regulation in response to IFN-I and IFN-II: a GAS and ISRE site within the STAT1 proximal promoter $(87,117,124)$; the upstream region of exon 1 containing an ISRE site (125); an IRF-binding element (IRF-E)/GAS/ IRF-E (IGI) motif at the intron 1/exon 2 boundary region (97): the recently identified distal ISRE and GAS-containing enhancer (5.5URR) (117).

The regulatory regions of the STAT2 and IRF9 genes were studied less extensive. According to Yan et al., the proximal promoter of STAT2 contains a functional ISRE, but no GAS (98). However, sequence alignment of the mouse and human genome sequence revealed conserved GAS-like and ISRE elements in the promoter that corresponded with IFN-I and IFN-II responsiveness $(87,117)$. Likewise, IRF9 harbors ISRE and GAS elements in its promoter, which are targets of ISGF3 and GAF in response to IFN-I or IFN-II $(87,117)$.

By using the K562 and 2fTGH ChIP-seq datasets, comparative ISGF3, GAF, and IRF1 binding patterns could also be observed for the STAT1, STAT2, and IRF9 genes (Figure 4). For all of these genes STAT1 and STAT2 binding as well as IRF9, was observed in the promoter (Figure 4; Table 1; STAT1: distal; STAT2 and IRF9: proximal) in a time-dependent manner (even after $24 \mathrm{~h}$ ) in $2 \mathrm{fTGH}$ and K562 IFN-I treated cells. This corresponds with the presence of an ISRE and a GAS element in close proximity in their promoters (Table 1). Under these conditions, IRF1 binding could be detected to STAT1 and STAT2 genes, and weakly to IRF9, which further substantiates the observation that STAT1, STAT2, and IRF9 are IRF1-targets (48). Binding of STAT1 and IRF1 to these promoter sites in the STAT1, STAT2, and IRF9 genes in response to IFN-II (Figure 4; K562 cells only), is in agreement 
with the presence of a functional GAS and ISRE element in mediating their IFN-II-induced transcription (97, 98, 117). Recently, a novel distal regulatory element was described positioned 5.5-kb upstream of the mouse Stat1 gene (117) with a similar ISRE and GAS composite structure as we observe here in the human gene (Figure 4: right peak; Table 1: distal). By association of STAT1 complexes, namely ISGF3 and GAF, this ISRE and GAS composite was proposed to mediate the autoregulation of STAT1 gene and its prolonged expression in response to IFN-I and IFN-II (117). The observations in Figure 4 are in agreement with this idea for STAT1 and confirm the presence of a similar ISRE and GAS organization for the human STAT1, STAT2, and IRF9 genes.

Closer examination identified co-binding of STAT1, STAT2, IRF9, and/or IRF1 in response to IFN-I and IFN-II in the different cell lines on a proximal site in the STAT1 promoter (Figure 4: left peak; Table 1: proximal), which likewise corresponded with the presence of putative ISRE site in the sequence under the peak, but no GAS could be recognized. Similarly, for the STAT2 gene a second STAT1, STAT2, and IRF9 binding peak could be observed in 2fTGH, but not (or very weak) in K562 (Figure 4: left peak), corresponding to a putative ISRE site (Table 1: intronic). The functionality of these sites in relation to STAT1 and STAT2 expression are currently not known. Basal binding of U-STAT1 to the STAT1, STAT2, and IRF9 promoters, as observed in the GM12878 cell line (Figure 4), correlates with IFN-independent expression of these genes (Figure 2) (55).

Together, the genomic organization and the binding pattern of ISGF3, GAF, and IRF1, predict the involvement of co-binding and provide an explanation for the positive feedback regulation in IFN-I and IFN-II activated expression of the STAT1, STAT2 and IRF9 and IRF1 genes.

\section{POSITIVE FEEDBACK REGULATION OF ISGF3 AND GAF COMPONENTS AND IRF1 IN PROGRESSION AND MAINTENANCE OF IFN-I AND IFN-II RESPONSES}

Based on the above, a picture emerges of a more complex and multifaceted mechanism of IFN-I and IFN-II-activated ISG regulation in which STAT1 and STAT2-containing ISGF3 and GAF-like complexes and IRF1 are recruited to individual or combined ISRE and GAS sites. This mechanism closely depends on positive feedback regulation of the STAT1, STAT2, IRF9, and IRF1 genes and facilitates long-term responses to IFN-I and IFN-II (Figure 5, central panel). In detail, IFN-I phosphorylates STAT1 and STAT2 and triggers the formation of STAT1 homodimers and STAT1-STAT2 heterodimers as well as ISGF3 that bind to ISRE and GAS sites in ISG promoters. IFN-II only phosphorylates STAT1 that forms homodimers to bind GAS sites. IFN-I and IFN-II also increase IRF1 expression through binding of STAT1 homodimers and STAT1-STAT2 heterodimers to a single GAS element in its promoter. On the one hand, positive feedback regulation of the STAT1, STAT2, IRF9, and IRF1 genes is achieved through binding of these complexes to combined ISRE and GAS-containing regulatory sequences (Figure 5, central panel). In IFN-I signaling ISGF3 and IRF1 bind the ISRE and STAT1 homodimers and possibly STAT1-STAT2 heterodimers the GAS. In IFN-II action, IRF1 binds the ISRE and STAT1 homodimers the GAS. This leads to increased STAT1, STAT2, IRF9, and IRF1 protein expression, further enhancing complex formation in an IFN-I and -II dependent manner (Figure 5, central panel). On the other hand, these complexes participate in the increased expression of other ISRE and/or GAS-containing ISGs (Figure 5, central panel).

Strong evidence exists that the IFN-dependent positive feedback regulation of STAT1, STAT2, IRF9, and IRF1 depends on phosphorylated ISGF3 and GAF components in the early response phase. For example, STAT1 KO U3A cells rescued with tyrosine 701 mutated STAT1 (Y to F) (53) or STAT1 KO mice overexpressing this STAT1 Y to F mutant (19), lack this positive feedback system. Likewise, STAT1 KO U3C cells, which also have low levels of STAT2, lack this positive feedback system and are unresponsive to IFN treatment and unable to protect against viral infection (30). However, STAT1 KO U3C cells overexpressing STAT2 regain the IFN-mediated positive feedback regulation of IRF9, IFN-I responsiveness and antiviral activity (30). Contrary, macrophages from STAT1 KO mice (32) as well as Huh-7.5 STAT1 mutant cells (12), which express normal STAT2 amounts, still exhibit this feedback system. This implies that a level of redundancy exists between the individual ISGF3 and GAF components, but also that a certain threshold level must be reached, to ensure positive feedback of their expression and progressive responses to IFNs.

The exact timely steps that take place during IFN-activated feedback regulation and the control of ISG transcription and long-term cellular responsiveness to IFN-I and IFN-II, is currently not clear.

One possible mechanism describes the involvement of an ISGF3-like complex composed of unphosphorylated STAT1 and 2 with IRF9 (named U-ISGF3) or U-GAF in long-term treated cells where increased levels of unphosporylated ISGF3 components highly dominate their phosphorylated counterparts $(10-12,107)$. As such, U-ISGF3 is proposed to switch with ISGF3 in time to drive prolonged expression of ISGs, including STAT1, STAT2, and IRF9, in response to IFN-I. Similarly, a switch of U-STAT1 with GAF can be envisioned, under these conditions (Figure 5, right panel). Interestingly, macrophages from STAT1 KO mice (32) as well as Huh-7.5 STAT1 mutant cells (12) exhibit increased expression of U-STAT2 and IRF9 upon long-term IFN-I treatment. Therefore, a possible role exists for U-STAT2/IRF9 in the durable expression of typical IFN-I activated ISGs (31). The expression of ISGF3 components is also highly increased in longterm IFN-II treated cells (11), providing the possibility for the formation of U-ISGF3 and U-GAF as well. Therefore, a regulatory role for U-ISGF3 and U-GAF in long-term IFN-II-treated cells might also exist (Figure 5, right panel), however, currently no experimental evidence exists for this scenario.

A second mechanism predicts the involvement of phosphorylated STAT1 and STAT2 together with IRF9 (as ISGF3 or STAT2/ IRF9) and phosphorylated STAT1 (as GAF) in positive feedback regulation and prolonged ISG expression in response to IFN-I and IFN-II (Figure 5, right panel). This is in agreement with the 


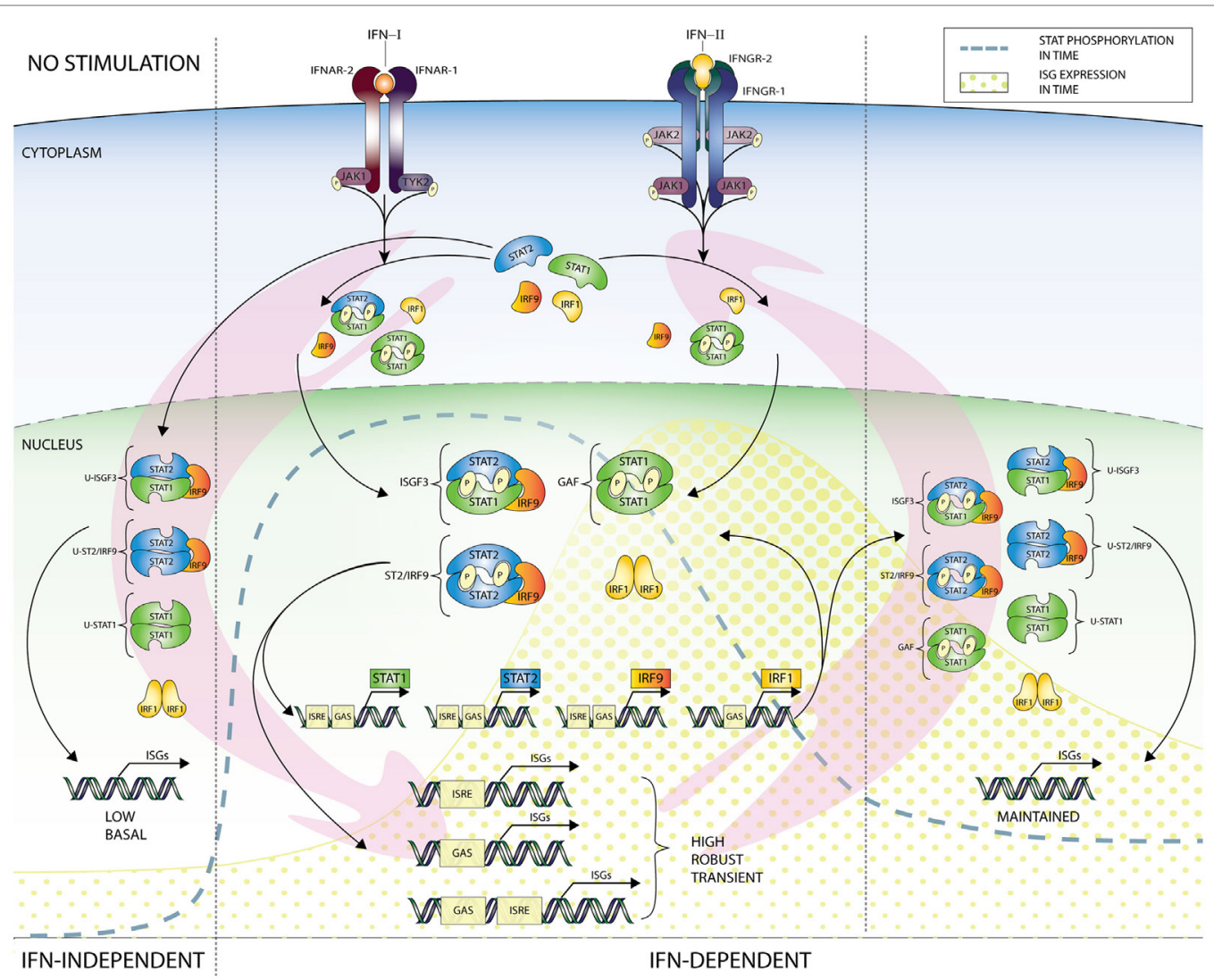

FIGURE 5 | Cooperation of unphosphorylated and phosphorylated ISGF3 and GAF components and IRF1 in the regulation of basal and IFN-induced transcription. In unstimulated cells, most of the known ISGs are expressed at low but detectable levels. Among these genes, there are examples of components (STATs and IRFs) of transcription factors, which in the absence of stimulation can combine in unphosphorylated complexes (U-ISGF3, U-STAT1, U-STAT2/IRF9, and IRF1) and drive the constitutive expression of ISGs. After cell stimulation with IFN type I or II, receptors dimerize and enable receptor-bound kinases (JAK1 and TYK2 for IFN-I or JAK1 and JAK2 for IFN-II) transphosphorylation and STAT protein recruitment. Next step is phosphorylation of STATs, which can now dimerize and with or without a partner (IRF9) create complexes - ISGF3, ST2/IRF9, and GAF, then translocate to the nucleus and start a high and robust but transient expression (yellow dotted graph) of GAS and/or ISRE-containing ISGs. This leads to, inter alia, rapid accumulation of newly synthesized STAT1, STAT2, IRF9, and IRF1 proteins in the cytoplasm, which can create new transcription factors in unphosphorylated form (U-ISGF3, U-STAT1, and U-STAT2/IRF9), and while level of phospho-proteins is dropping (dotted line) support or take over the role of phosphorylated complexes in sustaining the expression of ISGs. Important role in the circuit plays IRF1, which can occupy ISRE-containing genes and co-binds with other factors to modulate IFN-induced response, as well as basal ISG expression. As shown in the graph, responses to both types of IFN rely on common elements and events of the JAK-STAT signaling pathway and result in partially shared outcome. Thus, IFN-I and IFN-II induce a common as well as a unique set of genes. These facts clearly point to functional and biological overlap between IFN-I and IFN-II action and additionally to existence of mechanisms allowing cells to manage and adjust both responses. Abbreviations: IFN, interferon; STAT, signal transducer and activator of transcription; IRF, interferon regulatory factor; JAK, Janus kinase; TYK, tyrosine kinase; ISGF3, interferon-stimulated gene factor 3; GAF, $\gamma$-activated factor; ISRE, interferon-stimulated response element; GAS, $\gamma$-activated sequence; ISG, interferon-stimulated gene; P, phosphate; U, unphosphorylated form.

observation, that in many cell types levels of phosphorylated STAT1 and STAT2 drop from high to low upon long-term IFN-I and IFN-II treatment, being still detectable and maintained for several days $(11,112)$. At the same time, it correlates with the sustained expression of a large group of ISGs, including STAT1, STAT2, and IRF9, which follow the timely phosphorylation pattern of STAT1 and STAT2 (Figure 5, central panel). Likewise, the prolonged IFN-I activated phosphorylation of STAT2 in the absence of STAT1, strongly correlates with sustained ISG expression $(12,30,32)$. Also, a novel role of ISGF3 ${ }^{\text {II }}$, which consists of pSTAT1 together with U-STAT2 and IRF9, was proposed to be involved in long-term IFN-II signaling (11). Moreover, Yuasa et al., recently provided more insight into the autoregulatory mechanism of IFN-dependent STAT1 gene expression. They established that a novel distal regulatory element, 5.5URR, can mediate activation of the STAT1 gene, in an IFN-dependent manner. Indeed, by association of IFN-dependent STAT1 complexes, this enhancer was shown to physically associate with the STAT1 core promoter and regulate STAT1 gene expression in response to IFN-I and IFN-II (117). The ChIP-seq data investigation of K562 and $2 \mathrm{fTGH}$ cells (see above) are in agreement with this idea for STAT1 and provide a similar ISRE and GAS-dependent mechanism for the positive feedback regulation of STAT2 and IRF9 (Figure 4).

Third, as a STAT1-specific target gene IRF1 is highly induced in the early stages of IFN-I and IFN-II responses, while its levels are sustained upon long-term treatment following STAT1 phosphorylation (38). As such, IRF1 facilitates secondary responses to IFN-I and IFN-II in many cells. Among the target genes of 
IRF1 are STAT1, STAT2, and IRF9 and a diversity of other ISREcontaining ISGs, agreeing with a functional overlap with ISGF3 $(48,61)$. It also suggests the involvement of IRF1 in the feedback regulation of ISGF3 and GAF components as well as the sustained expression of typical antiviral ISGs (Figure 5, right panel).

Finally, a novel function for U-STAT1, U-STAT2, IRF9, and IRF1 is appearing in untreated cells as U-ISGF3, U-STAT2/IRF9, U-GAF, or IRF1 DNA-binding complexes to keep ISG expression at a low basal level $(55,75)$ (Figure 5, left panel). Since many ISGs represent key components of antiviral pathways, their basal expression is essential for the robust activation of these IFNactivated signaling pathways. Moreover, basal binding of these complexes could possibly mark the genome for rapid ISGF3, STAT2/IRF9, GAF, and/or IRF1 recruitment upon IFN-I and IFN-II treatment and prepare cells for a robust and effective response against invading pathogens.

\section{CONCLUSION}

According to the general paradigm, phosphorylation of STAT1 and STAT2 in response to IFN-I and/or IFN-II displays a robust and transient character. This is followed by a similar ISG expression pattern that decreases over time. However, numerous studies have shown that IFN signaling is much more complex and revealed that ISG expression patterns are globally sustained in response to both types of IFN (10-12). This sustained response relies on prolonged expression of the ISGF3 and GAF components STAT1, STAT2, and IRF9 and IRF1 as part of a positive feedback loop (Figure 5).

Together, this predicts the existence of a multifaceted intracellular amplifier circuit that depends on unphosphorylated and phosphorylated ISGF3 and GAF complexes and IRF1. In a combinatorial and timely fashion, these complexes mediate prolonged ISG expression and control cellular responsiveness to IFN-I and IFN-II (Figure 5). This proposed intracellular amplifier circuit also provides a molecular explanation for the existing overlap between IFN-I and IFN-II activated ISG expression.

The exact timely steps that take place during IFN-activated feedback regulation and the control of ISG transcription and

\section{REFERENCES}

1. Lindenmann J, Burke DC, Isaacs A. Studies on the production, mode of action and properties of interferon. Br J Exp Pathol (1957) 38(5):551-62.

2. Randall RE, Goodbourn S. Interferons and viruses: an interplay between induction, signalling, antiviral responses and virus countermeasures. J Gen Virol (2008) 89(Pt 1):1-47. doi:10.1099/vir.0.83391-0

3. Sadler AJ, Williams BR. Interferon-inducible antiviral effectors. Nat Rev Immunol (2008) 8(7):559-68. doi:10.1038/nri2314

4. Stark GR, Kerr IM, Williams BR, Silverman RH, Schreiber RD. How cells respond to interferons. Annu Rev Biochem (1998) 67:227-64. doi:10.1146/ annurev.biochem.67.1.227

5. Donnelly RP, Kotenko SV. Interferon-lambda: a new addition to an old family. J Interferon Cytokine Res (2010) 30(8):555-64. doi:10.1089/jir.2010.0078

6. O'Brien TR, Prokunina-Olsson L, Donnelly RP. IFN-lambda4: the paradoxical new member of the interferon lambda family. J Interferon Cytokine Res (2014) 34(11):829-38. doi:10.1089/jir.2013.0136

7. Ivashkiv LB, Donlin LT. Regulation of type I interferon responses. Nat Rev Immunol (2014) 14(1):36-49. doi:10.1038/nri3581 long-term cellular responsiveness to IFN-I and IFN-II, are still not clear. The same holds true for the distinct functions of unphosporylated ISGF3 and GAF complexes in relation to their phosphorylated counterparts, especially in the transition from early to sustained IFN actions. For this, more detailed time-dependent genome-wide expression and chromatin interaction studies are required in wild type as compared to knockout back grounds of ISGF3 and GAF components and IRF1. This will allow discrimination between IFN-independent and IFN-dependent conditions, and between U-STAT and pSTAT regulatory functions and binding characteristics on promoter proximal and distal ISRE and GAS-containing sites. Moreover, it will provide insight in the role of the different components in the complex IFN-independent and IFN-dependent transcriptional control mechanisms involved in early and sustained ISG transcription.

\section{AUTHOR CONTRIBUTIONS}

AM was involved in concept development and writing and editing the manuscript, analyzed published ChIP-seq data, and designed the figures and table. KB generated novel ChIP-seq data and provided critical feedback on the manuscript. JW participated in development of the concept and critically evaluated and edited the manuscript. HB developed the concept and was involved in writing and editing the manuscript and coordinated input from all co-authors.

\section{FUNDING}

This work was supported by the Polish Ministry of Science and Higher Education (http://www.ncn.gov.pl/) [OPUS grant numbers: UMO-2013/11/B/NZ2/02569 (HAR.B.), UMO 2016/23/B/ NZ2/00623 (HAR.B.) and ETIUDA grant number UMO2015/16/T/NZ3/00054 (KB)], the KNOW RNA Research Centre in Poznan (http://know-rna.amu.edu.pl/en/) [grant number 01/ KNOW2/2014]; the National Multidisciplinary Laboratory of Functional Nanomaterials NanoFun project (http://www.nanofun.edu.pl/) [grant number POIG.02.02.00-00-025/09 (JW)].

8. Kroger A, Koster M, Schroeder K, Hauser H, Mueller PP. Activities of IRF-1. J Interferon Cytokine Res (2002) 22(1):5-14. doi:10.1089/107999002753452610

9. Pine R, Decker T, Kessler DS, Levy DE, Darnell JE Jr. Purification and cloning of interferon-stimulated gene factor 2 (ISGF2): ISGF2 (IRF-1) can bind to the promoters of both beta interferon- and interferon-stimulated genes but is not a primary transcriptional activator of either. Mol Cell Biol (1990) 10(6):2448-57. doi:10.1128/MCB.10.6.2448

10. Cheon H, Holvey-Bates EG, Schoggins JW, Forster S, Hertzog P, Imanaka N, et al. IFNbeta-dependent increases in STAT1, STAT2, and IRF9 mediate resistance to viruses and DNA damage. EMBO J (2013) 32(20):2751-63. doi:10.1038/emboj.2013.203

11. Morrow AN, Schmeisser H, Tsuno T, Zoon KC. A novel role for IFNstimulated gene factor 3II in IFN-gamma signaling and induction of antiviral activity in human cells. J Immunol (2011) 186(3):1685-93. doi:10.4049/ jimmunol.1001359

12. Yamauchi S, Takeuchi K, Chihara K, Honjoh C, Kato Y, Yoshiki H, et al. STAT1 is essential for the inhibition of hepatitis $\mathrm{C}$ virus replication by interferon-lambda but not by interferon-alpha. Sci Rep (2016) 6:38336. doi: $10.1038 /$ srep38336 
13. Levy DE, Darnell JE Jr. Stats: transcriptional control and biological impact. Nat Rev Mol Cell Biol (2002) 3(9):651-62. doi:10.1038/nrm909

14. Platanias LC. Introduction: interferon signals: what is classical and what is nonclassical? J Interferon Cytokine Res (2005) 25(12):732. doi:10.1089/ jir.2005.25.732

15. Wesoly J, Szweykowska-Kulinska Z, Bluyssen HA. STAT activation and differential complex formation dictate selectivity of interferon responses. Acta Biochim Pol (2007) 54(1):27-38.

16. Schneider WM, Chevillotte MD, Rice CM. Interferon-stimulated genes: a complex web of host defenses. Annu Rev Immunol (2014) 32:513-45. doi:10.1146/annurev-immunol-032713-120231

17. Banninger G, Reich NC. STAT2 nuclear trafficking. J Biol Chem (2004) 279(38):39199-206. doi:10.1074/jbc.M400815200

18. Levy DE, Kessler DS, Pine R, Darnell JE Jr. Cytoplasmic activation of ISGF3, the positive regulator of interferon-alpha-stimulated transcription, reconstituted in vitro. Genes Dev (1989) 3(9):1362-71. doi:10.1101/gad. 3.9.1362

19. Majoros A, Platanitis E, Kernbauer-Holzl E, Rosebrock F, Muller M, Decker T. Canonical and non-canonical aspects of JAK-STAT signaling: lessons from interferons for cytokine responses. Front Immunol (2017) 8:29. doi:10.3389/ fimmu.2017.00029

20. Yoshimura A, Naka T, Kubo M. SOCS proteins, cytokine signalling and immune regulation. Nat Rev Immunol (2007) 7(6):454-65. doi:10.1038/ nri2093

21. Bluyssen HA, Levy DE. Stat2 is a transcriptional activator that requires sequence-specific contacts provided by stat 1 and 48 for stable interaction with DNA. J Biol Chem (1997) 272(7):4600-5. doi:10.1074/jbc.272.7.4600

22. Kraus TA, Lau JF, Parisien JP, Horvath CM. A hybrid IRF9-STAT2 protein recapitulates interferon-stimulated gene expression and antiviral response. J Biol Chem (2003) 278(15):13033-8. doi:10.1074/jbc.M212972200

23. Poat B, Hazari S, Chandra PK, Gunduz F, Alvarez X, Balart LA, et al. Intracellular expression of IRF9 Stat fusion protein overcomes the defective Jak-Stat signaling and inhibits HCV RNA replication. Virol J (2010) 7:265. doi:10.1186/1743-422X-7-265

24. Bluyssen AR, Durbin JE, Levy DE. ISGF3 gamma p48, a specificity switch for interferon activated transcription factors. Cytokine Growth Factor Rev (1996) 7(1):11-7. doi:10.1016/1359-6101(96)00005-6

25. Fink K, Grandvaux N. STAT2 and IRF9: beyond ISGF3. JAKSTAT (2013) 2(4):e27521. doi:10.4161/jkst.27521

26. Steen HC, Gamero AM. The role of signal transducer and activator of transcription-2 in the interferon response. J Interferon Cytokine Res (2012) 32(3):103-10. doi:10.1089/jir.2011.0099

27. Steen HC, Gamero AM. STAT2 phosphorylation and signaling. JAKSTAT (2013) 2(4):e25790. doi:10.4161/jkst.25790

28. Ghislain JJ, Wong $T$, Nguyen $M$, Fish EN. The interferon-inducible Stat2:Stat 1 heterodimer preferentially binds in vitro to a consensus element found in the promoters of a subset of interferon-stimulated genes. J Interferon Cytokine Res (2001) 21(6):379-88. doi:10.1089/107999001750277853

29. Gupta S, Jiang M, Pernis AB. IFN-alpha activates Stat6 and leads to the formation of Stat2:Stat6 complexes in B cells. J Immunol (1999) 163(7): $3834-41$.

30. Blaszczyk K, Olejnik A, Nowicka H, Ozgyin L, Chen YL, Chmielewski S, et al. STAT2/IRF9 directs a prolonged ISGF3-like transcriptional response and antiviral activity in the absence of STAT1. Biochem J (2015) 466(3):511-24. doi:10.1042/BJ20140644

31. Lou YJ, Pan XR, Jia PM, Li D, Xiao S, Zhang ZL, et al. IRF-9/STAT2 [corrected] functional interaction drives retinoic acid-induced gene $G$ expression independently of STAT1. Cancer Res (2009) 69(8):3673-80. doi:10.1158/0008-5472.CAN-08-4922

32. Abdul-Sater AA, Majoros A, Plumlee CR, Perry S, Gu AD, Lee C, et al. Different STAT transcription complexes drive early and delayed responses to type I IFNs. J Immunol (2015) 195(1):210-6. doi:10.4049/jimmunol.1401139

33. Fenner JE, Starr R, Cornish AL, Zhang JG, Metcalf D, Schreiber RD, et al. Suppressor of cytokine signaling 1 regulates the immune response to infection by a unique inhibition of type I interferon activity. Nat Immunol (2006) 7(1):33-9. doi:10.1038/ni1287

34. Didcock L, Young DF, Goodbourn S, Randall RE. The V protein of simian virus 5 inhibits interferon signalling by targeting STAT1 for proteasomemediated degradation. J Virol (1999) 73(12):9928-33.
35. Ramachandran A, Horvath CM. Paramyxovirus disruption of interferon signal transduction: STATus report. J Interferon Cytokine Res (2009) 29(9): 531-7. doi:10.1089/jir.2009.0070

36. Takeuchi K, Komatsu T, Yokoo J, Kato A, Shioda T, Nagai Y, et al. Sendai virus C protein physically associates with Stat1. Genes Cells (2001) 6(6):545-57. doi:10.1046/j.1365-2443.2001.00442.x

37. Reich NC. STATs get their move on. JAKSTAT (2013) 2(4):e27080. doi:10.4161/ jkst. 27080

38. Stewart MD, Choi Y, Johnson GA, Yu-Lee LY, Bazer FW, Spencer TE. Roles of Stat1, Stat2, and interferon regulatory factor-9 (IRF-9) in interferon tau regulation of IRF-1. Biol Reprod (2002) 66(2):393-400. doi:10.1095/ biolreprod66.2.393

39. Horvath CM, Wen Z, Darnell JE Jr. A STAT protein domain that determines DNA sequence recognition suggests a novel DNA-binding domain. Genes $\operatorname{Dev}$ (1995) 9(8):984-94. doi:10.1101/gad.9.8.984

40. Decker T, Kovarik P, Meinke A. GAS elements: a few nucleotides with a major impact on cytokine-induced gene expression. J Interferon Cytokine Res (1997) 17(3):121-34. doi:10.1089/jir.1997.17.121

41. Borden EC, Sen GC, Uze G, Silverman RH, Ransohoff RM, Foster GR, et al. Interferons at age 50: past, current and future impact on biomedicine. Nat Rev Drug Discov (2007) 6(12):975-90. doi:10.1038/nrd2422

42. Brass AL, Huang IC, Benita Y, John SP, Krishnan MN, Feeley EM, et al. The IFITM proteins mediate cellular resistance to influenza A H1N1 virus, West Nile virus, and dengue virus. Cell (2009) 139(7):1243-54. doi:10.1016/j. cell.2009.12.017

43. Dong B, Zhou Q, Zhao J, Zhou A, Harty RN, Bose S, et al. Phospholipid scramblase 1 potentiates the antiviral activity of interferon. J Virol (2004) 78(17):8983-93. doi:10.1128/JVI.78.17.8983-8993.2004

44. Itsui Y, Sakamoto N, Kakinuma S, Nakagawa M, Sekine-Osajima Y, TasakaFujita M, et al. Antiviral effects of the interferon-induced protein guanylate binding protein 1 and its interaction with the hepatitis C virus NS5B protein. Hepatology (2009) 50(6):1727-37. doi:10.1002/hep.23195

45. Miyashita M, Oshiumi H, Matsumoto M, Seya T. DDX60, a DEXD/H box helicase, is a novel antiviral factor promoting RIG-I-like receptor-mediated signaling. Mol Cell Biol (2011) 31(18):3802-19. doi:10.1128/MCB.01368-10

46. Neil SJ, Zang T, Bieniasz PD. Tetherin inhibits retrovirus release and is antagonized by HIV-1 Vpu. Nature (2008) 451(7177):425-30. doi:10.1038/ nature 06553

47. Schmeisser H, Mejido J, Balinsky CA, Morrow AN, Clark CR, Zhao T, et al. Identification of alpha interferon-induced genes associated with antiviral activity in Daudi cells and characterization of IFIT3 as a novel antiviral gene. J Virol (2010) 84(20):10671-80. doi:10.1128/JVI.00818-10

48. Schoggins JW, Wilson SJ, Panis M, Murphy MY, Jones CT, Bieniasz P, et al. A diverse range of gene products are effectors of the type I interferon antiviral response. Nature (2011) 472(7344):481-5. doi:10.1038/nature09907

49. Tang Y, Zhong G, Zhu L, Liu X, Shan Y, Feng H, et al. Herc5 attenuates influenza A virus by catalyzing ISGylation of viral NS1 protein. J Immunol (2010) 184(10):5777-90. doi:10.4049/jimmunol.0903588

50. Oudshoorn D, Versteeg GA, Kikkert M. Regulation of the innate immune system by ubiquitin and ubiquitin-like modifiers. Cytokine Growth Factor $\operatorname{Rev}(2012)$ 23(6):273-82. doi:10.1016/j.cytogfr.2012.08.003

51. Tan J, Qiao W, Wang J, Xu F, Li Y, Zhou J, et al. IFP35 is involved in the antiviral function of interferon by association with the viral tas transactivator of bovine foamy virus. J Virol (2008) 82(9):4275-83. doi:10.1128/JVI.02249-07

52. Meyer T, Vinkemeier U. Nucleocytoplasmic shuttling of STAT transcription factors. Eur J Biochem (2004) 271(23-24):4606-12. doi:10.1111/j.1432-1033. 2004.04423.x

53. Cheon H, Stark GR. Unphosphorylated STAT1 prolongs the expression of interferon-induced immune regulatory genes. Proc Natl Acad Sci U S A (2009) 106(23):9373-8. doi:10.1073/pnas.0903487106

54. Yao K, Chen Q, Wu Y, Liu F, Chen X, Zhang Y. Unphosphorylated STAT1 represses apoptosis in macrophages during Mycobacterium tuberculosis infection. J Cell Sci (2017) 130(10):1740-51. doi:10.1242/jcs.200659

55. Wang W, Yin Y, Xu L, Su J, Huang F, Wang Y, et al. Unphosphorylated ISGF3 drives constitutive expression of interferon-stimulated genes to protect against viral infections. Sci Signal (2017) 10(476). doi:10.1126/ scisignal.aah 4248

56. Chatterjee-Kishore M, Wright KL, Ting JP, Stark GR. How Stat1 mediates constitutive gene expression: a complex of unphosphorylated Statl and IRF1 
supports transcription of the LMP2 gene. EMBO J (2000) 19(15):4111-22. doi:10.1093/emboj/19.15.4111

57. Meyer T, Marg A, Lemke P, Wiesner B, Vinkemeier U. DNA binding controls inactivation and nuclear accumulation of the transcription factor Stat1. Genes Dev (2003) 17(16):1992-2005. doi:10.1101/gad.268003

58. Barnes B, Lubyova B, Pitha PM. On the role of IRF in host defense. J Interferon Cytokine Res (2002) 22(1):59-71. doi:10.1089/107999002753452665

59. Taniguchi T, Ogasawara K, Takaoka A, Tanaka N. IRF family of transcription factors as regulators of host defense. Annu Rev Immunol (2001) 19:623-55. doi:10.1146/annurev.immunol.19.1.623

60. Schaper F, Kirchhoff S, Posern G, Koster M, Oumard A, Sharf R, et al. Functional domains of interferon regulatory factor I (IRF-1). Biochem $J$ (1998) 335(Pt 1):147-57. doi:10.1042/bj3350147

61. Kimura T, Kadokawa Y, Harada H, Matsumoto M, Sato M, Kashiwazaki Y, et al. Essential and non-redundant roles of p48 (ISGF3 gamma) and IRF-1 in both type I and type II interferon responses, as revealed by gene targeting studies. Genes Cells (1996) 1(1):115-24. doi:10.1046/j.1365-2443.1996.08008.x

62. Haque SJ, Williams BR. Identification and characterization of an interferon (IFN)-stimulated response element-IFN-stimulated gene factor 3-independent signaling pathway for IFN-alpha. J Biol Chem (1994) 269(30):19523-9.

63. Blaszczyk K, Nowicka H, Kostyrko K, Antonczyk A, Wesoly J, Bluyssen HA. The unique role of STAT2 in constitutive and IFN-induced transcription and antiviral responses. Cytokine Growth Factor Rev (2016) 29:71-81. doi:10.1016/j.cytogfr.2016.02.010

64. Brierley MM, Fish EN. Functional relevance of the conserved DNA-binding domain of STAT2. J Biol Chem (2005) 280(13):13029-36. doi:10.1074/jbc. M500426200

65. Brierley MM, Marchington KL, Jurisica I, Fish EN. Identification of GAS-dependent interferon-sensitive target genes whose transcription is STAT2-dependent but ISGF3-independent. FEBS J (2006) 273(7):1569-81. doi:10.1111/j.1742-4658.2006.05176.x

66. Ghislain JJ, Fish EN. Application of genomic DNA affinity chromatography identifies multiple interferon-alpha-regulated Stat2 complexes. J Biol Chem (1996) 271(21):12408-13. doi:10.1074/jbc.271.21.12408

67. Li X, Leung S, Qureshi S, Darnell JE Jr, Stark GR. Formation of STAT1-STAT2 heterodimers and their role in the activation of IRF-1 gene transcription by interferon-alpha. J Biol Chem (1996) 271(10):5790-4. doi:10.1074/ jbc.271.10.5790

68. Xu L, Zhou X, Wang W, Wang Y, Yin Y, Laan LJ, et al. IFN regulatory factor 1 restricts hepatitis E virus replication by activating STAT1 to induce antiviral IFN-stimulated genes. FASEB J (2016) 30(10):3352-67. doi:10.1096/ f. $201600356 \mathrm{R}$

69. Imam AM, Ackrill AM, Dale TC, Kerr IM, Stark GR. Transcription factors induced by interferons alpha and gamma. Nucleic Acids Res (1990) 18(22):6573-80. doi:10.1093/nar/18.22.6573

70. Parrington J, Rogers NC, Gewert DR, Pine R, Veals SA, Levy DE, et al. The interferon-stimulable response elements of two human genes detect overlapping sets of transcription factors. Eur J Biochem (1993) 214(3): 617-26. doi:10.1111/j.1432-1033.1993.tb17961.x

71. Morris AC, Beresford GW, Mooney MR, Boss JM. Kinetics of a gamma interferon response: expression and assembly of CIITA promoter IV and inhibition by methylation. Mol Cell Biol (2002) 22(13):4781-91. doi:10.1128/ МСB.22.13.4781-4791.2002

72. Ramsauer K, Farlik M, Zupkovitz G, Seiser C, Kroger A, Hauser H, et al. Distinct modes of action applied by transcription factors STAT1 and IRF1 to initiate transcription of the IFN-gamma-inducible gbp2 gene. Proc Natl Acad Sci U S A (2007) 104(8):2849-54. doi:10.1073/pnas.0610944104

73. Kumatori A, Yang D, Suzuki S, Nakamura M. Cooperation of STAT-1 and IRF-1 in interferon-gamma-induced transcription of the gp91(phox) gene. J Biol Chem (2002) 277(11):9103-11. doi:10.1074/jbc.M109803200

74. Xie D, Boyle AP, Wu L, Zhai J, Kawli T, Snyder M. Dynamic trans-acting factor colocalization in human cells. Cell (2013) 155(3):713-24. doi:10.1016/ j.cell.2013.09.043

75. Abou El Hassan M, Huang K, Eswara MB, Xu Z, Yu T, Aubry A, et al. Properties of STAT1 and IRF1 enhancers and the influence of SNPs. BMC Mol Biol (2017) 18(1):6. doi:10.1186/s12867-017-0084-1

76. Dupuis S, Dargemont C, Fieschi C, Thomassin N, Rosenzweig S, Harris J, et al. Impairment of mycobacterial but not viral immunity by a germline human STAT1 mutation. Science (2001) 293(5528):300-3. doi:10.1126/ science. 1061154

77. Sampaio EP, Bax HI, Hsu AP, Kristosturyan E, Pechacek J, Chandrasekaran P, et al. A novel STAT1 mutation associated with disseminated mycobacterial disease. J Clin Immunol (2012) 32(4):681-9. doi:10.1007/s10875-012-9659-2

78. Chapgier A, Boisson-Dupuis S, Jouanguy E, Vogt G, Feinberg J, ProchnickaChalufour A, et al. Novel STAT1 alleles in otherwise healthy patients with mycobacterial disease. PLoS Genet (2006) 2(8):e131. doi:10.1371/journal. pgen.0020131

79. Dupuis S, Jouanguy E, Al-Hajjar S, Fieschi C, Al-Mohsen IZ, Al-Jumaah S, et al. Impaired response to interferon-alpha/beta and lethal viral disease in human STAT1 deficiency. Nat Genet (2003) 33(3):388-91. doi:10.1038/ ng1097

80. Toubiana J, Okada S, Hiller J, Oleastro M, Lagos Gomez M, Aldave Becerra JC, et al. Heterozygous STAT1 gain-of-function mutations underlie an unexpectedly broad clinical phenotype. Blood (2016) 127(25):3154-64. doi:10.1182/ blood-2015-11-679902

81. Moens L, Van Eyck L, Jochmans D, Mitera T, Frans G, Bossuyt X, et al. A novel kindred with inherited STAT2 deficiency and severe viral illness. J Allergy Clin Immunol (2017) 139(6):1995-7.e9. doi:10.1016/j.jaci.2016.10.033

82. Hambleton S, Goodbourn S, Young DF, Dickinson P, Mohamad SM, Valappil M, et al. STAT2 deficiency and susceptibility to viral illness in humans. Proc Natl Acad Sci U S A (2013) 110(8):3053-8. doi:10.1073/ pnas. 1220098110

83. Au-Yeung N, Mandhana R, Horvath CM. Transcriptional regulation by STAT1 and STAT2 in the interferon JAK-STAT pathway. JAKSTAT (2013) 2(3):e23931. doi:10.4161/jkst.23931

84. Pellegrini S, Schindler C. Early events in signalling by interferons. Trends Biochem Sci (1993) 18(9):338-42. doi:10.1016/0968-0004(93)90070-4

85. Bluyssen HA, Vlietstra RJ, Faber PW, Smit EM, Hagemeijer A, Trapman J. Structure, chromosome localization, and regulation of expression of the interferon-regulated mouse Ifi54/Ifi56 gene family. Genomics (1994) 24(1): 137-48. doi:10.1006/geno.1994.1591

86. Ronni T, Matikainen S, Lehtonen A, Palvimo J, Dellis J, Van Eylen F, et al. The proximal interferon-stimulated response elements are essential for interferon responsiveness: a promoter analysis of the antiviral MxA gene. J Interferon Cytokine Res (1998) 18(9):773-81. doi:10.1089/jir.1998.18.773

87. Testoni B, Vollenkle C, Guerrieri F, Gerbal-Chaloin S, Blandino G, Levrero M. Chromatin dynamics of gene activation and repression in response to interferon alpha (IFN(alpha)) reveal new roles for phosphorylated and unphosphorylated forms of the transcription factor STAT2. J Biol Chem (2011) 286(23):20217-27. doi:10.1074/jbc.M111.231068

88. Darnell JE Jr, Kerr IM, Stark GR. Jak-STAT pathways and transcriptional activation in response to IFNs and other extracellular signaling proteins. Science (1994) 264(5164):1415-21. doi:10.1126/science.8197455

89. Wang Q, Floyd-Smith G. The p69/71 2-5A synthetase promoter contains multiple regulatory elements required for interferon-alpha-induced expression. DNA Cell Biol (1997) 16(12):1385-94. doi:10.1089/dna.1997. 16.1385

90. Rebouillat D, Hovnanian A, David G, Hovanessian AG, Williams BR. Characterization of the gene encoding the $100-\mathrm{kDa}$ form of human 2,5 ' oligoadenylate synthetase. Genomics (2000) 70(2):232-40. doi:10.1006/ geno. 2000.6382

91. Wang N, Dong Q, Li J, Jangra RK, Fan M, Brasier AR, et al. Viral induction of the zinc finger antiviral protein is IRF3-dependent but NF-kappaB-independent. J Biol Chem (2010) 285(9):6080-90. doi:10.1074/jbc.M109.054486

92. Gongora C, Degols G, Espert L, Hua TD, Mechti N. A unique ISRE, in the TATA-less human Isg20 promoter, confers IRF-1-mediated responsiveness to both interferon type I and type II. Nucleic Acids Res (2000) 28(12):2333-41. doi:10.1093/nar/28.12.2333

93. Qin H, Roberts KL, Niyongere SA, Cong Y, Elson CO, Benveniste EN. Molecular mechanism of lipopolysaccharide-induced SOCS-3 gene expression in macrophages and microglia. J Immunol (2007) 179(9):5966-76. doi:10.4049/jimmunol.179.9.5966

94. Tessitore A, Pastore L, Rispoli A, Cilenti L, Toniato E, Flati V, et al. Two gamma-interferon-activation sites (GAS) on the promoter of the human intercellular adhesion molecule (ICAM-1) gene are required for induction of transcription by IFN-gamma. Eur J Biochem (1998) 258(3):968-75. doi:10.1046/j.1432-1327.1998.2580968.x 
95. Pine R, Canova A, Schindler C. Tyrosine phosphorylated p91 binds to a single element in the ISGF2/IRF-1 promoter to mediate induction by IFN alpha and IFN gamma, and is likely to autoregulate the p91 gene. EMBO J (1994) 13(1):158-67.

96. Harada H, Takahashi E, Itoh S, Harada K, Hori TA, Taniguchi T. Structure and regulation of the human interferon regulatory factor 1 (IRF-1) and IRF-2 genes: implications for a gene network in the interferon system. Mol Cell Biol (1994) 14(2):1500-9. doi:10.1128/MCB.14.2.1500

97. Wong LH, Sim H, Chatterjee-Kishore M, Hatzinisiriou I, Devenish RJ, Stark G, et al. Isolation and characterization of a human STAT1 gene regulatory element. Inducibility by interferon (IFN) types I and II and role of IFN regulatory factor-1. J Biol Chem (2002) 277(22):19408-17. doi:10.1074/ jbc.M111302200

98. Yan R, Qureshi S, Zhong Z, Wen Z, Darnell JE Jr. The genomic structure of the STAT genes: multiple exons in coincident sites in Statl and Stat2. Nucleic Acids Res (1995) 23(3):459-63. doi:10.1093/nar/23.3.459

99. Ogony J, Choi HJ, Lui A, Cristofanilli M, Lewis-Wambi J. Interferon-induced transmembrane protein 1 (IFITM1) overexpression enhances the aggressive phenotype of SUM149 inflammatory breast cancer cells in a signal transducer and activator of transcription 2 (STAT2)-dependent manner. Breast Cancer Res (2016) 18(1):25. doi:10.1186/s13058-016-0683-7

100. Ohtomo T, Sugamata Y, Ozaki Y, Ono K, Yoshimura Y, Kawai S, et al. Molecular cloning and characterization of a surface antigen preferentially overexpressed on multiple myeloma cells. Biochem Biophys Res Commun (1999) 258(3):583-91. doi:10.1006/bbrc.1999.0683

101. Min W, Pober JS, Johnson DR. Interferon induction of TAP1: the phosphatase SHP-1 regulates crossover between the IFN-alpha/beta and the IFN-gamma signal-transduction pathways. Circ Res (1998) 83(8):815-23. doi:10.1161/ 01.RES.83.8.815

102. Yang W, Tan J, Liu R, Cui X, Ma Q, Geng Y, et al. Interferon-gamma upregulates expression of IFP35 gene in HeLa cells via interferon regulatory factor-1. PLoS One (2012) 7(12):e50932. doi:10.1371/journal.pone.0050932

103. Lefebvre S, Berrih-Aknin S, Adrian F, Moreau P, Poea S, Gourand L, et al. A specific interferon (IFN)-stimulated response element of the distal HLA-G promoter binds IFN-regulatory factor 1 and mediates enhancement of this nonclassical class I gene by IFN-beta. J Biol Chem (2001) 276(9):6133-9. doi:10.1074/jbc.M008496200

104. Decker T, Lew DJ, Cheng YS, Levy DE, Darnell JE Jr. Interactions of alpha- and gamma-interferon in the transcriptional regulation of the gene encoding a guanylate-binding protein. EMBO J (1989) 8(7):2009-14.

105. Lew DJ, Decker T, Strehlow I, Darnell JE. Overlapping elements in the guanylate-binding protein gene promoter mediate transcriptional induction by alpha and gamma interferons. Mol Cell Biol (1991) 11(1):182-91. doi:10.1128/MCB.11.1.182

106. Decker T, Lew DJ, Mirkovitch J, Darnell JE Jr. Cytoplasmic activation of GAF, an IFN-gamma-regulated DNA-binding factor. EMBO J (1991) 10(4):927-32.

107. Trilling M, Bellora N, Rutkowski AJ, de Graaf M, Dickinson P, Robertson K, et al. Deciphering the modulation of gene expression by type I and II interferons combining 4sU-tagging, translational arrest and in silico promoter analysis. Nucleic Acids Res (2013) 41(17):8107-25. doi:10.1093/nar/gkt589

108. Vinkemeier U. Getting the message across, STAT! Design principles of a molecular signaling circuit. J Cell Biol (2004) 167(2):197-201. doi:10.1083/ jcb.200407163

109. Begitt A, Droescher M, Meyer T, Schmid CD, Baker M, Antunes F, et al. STAT1-cooperative DNA binding distinguishes type 1 from type 2 interferon signaling. Nat Immunol (2014) 15(2):168-76. doi:10.1038/ni.2794

110. Hartman SE, Bertone P, Nath AK, Royce TE, Gerstein M, Weissman S, et al. Global changes in STAT target selection and transcription regulation upon interferon treatments. Genes Dev (2005) 19(24):2953-68. doi:10.1101/ gad.1371305
111. Consortium EP. An integrated encyclopedia of DNA elements in the human genome. Nature (2012) 489(7414):57-74. doi:10.1038/nature11247

112. Megger DA, Philipp J, Le-Trilling VTK, Sitek B, Trilling M. Deciphering of the human interferon-regulated proteome by mass spectrometry-based quantitative analysis reveals extent and dynamics of protein induction and repression. Front Immunol (2017) 8:1139. doi:10.3389/fimmu.2017.01139

113. Abou El Hassan M, Bremner R. A rapid simple approach to quantify chromosome conformation capture. Nucleic Acids Res (2009) 37(5):e35. doi:10.1093/ nar/gkp028

114. Harismendy O, Notani D, Song X, Rahim NG, Tanasa B, Heintzman N, et al. 9p21 DNA variants associated with coronary artery disease impair interferon-gamma signalling response. Nature (2011) 470(7333):264-8. doi:10.1038/ nature 09753

115. Ni Z, Abou El Hassan M, Xu Z, Yu T, Bremner R. The chromatin-remodeling enzyme BRG1 coordinates CIITA induction through many interdependent distal enhancers. Nat Immunol (2008) 9(7):785-93. doi:10.1038/ni.1619

116. Li P, Shi ML, Shen WL, Zhang Z, Xie DJ, Zhang XY, et al. Coordinated regulation of IFITM1, 2 and 3 genes by an IFN-responsive enhancer through longrange chromatin interactions. Biochim Biophys Acta (2017) 1860(8):885-93. doi:10.1016/j.bbagrm.2017.05.003

117. Yuasa K, Hijikata T. Distal regulatory element of the STAT1 gene potentially mediates positive feedback control of STAT1 expression. Genes Cells (2016) 21(1):25-40. doi:10.1111/gtc.12316

118. Yuasa K, Takeda S, Hijikata T. A conserved regulatory element located far downstream of the gls locus modulates gls expression through chromatin loop formation during myogenesis. FEBS Lett (2012) 586(19):3464-70. doi:10.1016/j.febslet.2012.07.074

119. Frontini M, Vijayakumar M, Garvin A, Clarke N. A ChIP-chip approach reveals a novel role for transcription factor IRF1 in the DNA damage response. Nucleic Acids Res (2009) 37(4):1073-85. doi:10.1093/nar/gkn1051

120. Rettino A, Clarke NM. Genome-wide identification of IRF1 binding sites reveals extensive occupancy at cell death associated genes. J Carcinog Mutagen (2013) S6:009. doi:10.4172/2157-2518.S6-009

121. Shi L, Perin JC, Leipzig J, Zhang Z, Sullivan KE. Genome-wide analysis of interferon regulatory factor I binding in primary human monocytes. Gene (2011) 487(1):21-8. doi:10.1016/j.gene.2011.07.004

122. White LC, Wright KL, Felix NJ, Ruffner H, Reis LF, Pine R, et al. Regulation of LMP2 and TAP1 genes by IRF-1 explains the paucity of CD8+ T cells in IRF-1-/- mice. Immunity (1996) 5(4):365-76. doi:10.1016/S1074-7613(00) 80262-9

123. Heintzman ND, Hon GC, Hawkins RD, Kheradpour P, Stark A, Harp LF, et al. Histone modifications at human enhancers reflect global cell-type-specific gene expression. Nature (2009) 459(7243):108-12. doi:10.1038/nature07829

124. He F, Ge W, Martinowich K, Becker-Catania S, Coskun V, Zhu W, et al. A positive autoregulatory loop of Jak-STAT signaling controls the onset of astrogliogenesis. Nat Neurosci (2005) 8(5):616-25. doi:10.1038/nn1440

125. Amalraj J, Cutler SJ, Ghazawi I, Boyle GM, Ralph SJ. REST negatively and ISGF3 positively regulate the human STAT1 gene in melanoma. Mol Cancer Ther (2013) 12(7):1288-98. doi:10.1158/1535-7163.MCT-12-0923

Conflict of Interest Statement: The authors declare that the research was conducted in the absence of any commercial or financial relationships that could be construed as a potential conflict of interest.

Copyright (c) 2018 Michalska, Blaszczyk, Wesoly and Bluyssen. This is an open-access article distributed under the terms of the Creative Commons Attribution License (CC BY). The use, distribution or reproduction in other forums is permitted, provided the original author(s) and the copyright owner are credited and that the original publication in this journal is cited, in accordance with accepted academic practice. No use, distribution or reproduction is permitted which does not comply with these terms. 